\title{
Circulating tumor DNA predicts neoadjuvant immunotherapy efficacy and recurrence-free survival in surgical non-small cell lung cancer patients
}

\author{
Dongsheng Yue ${ }^{1 \#}$, Weiran Liu ${ }^{2 \#}$, Chen Chen ${ }^{1 \#}$, Tao Zhang ${ }^{3 \#}$, Yuchen Ma ${ }^{1}$, Longgang Cui ${ }^{4}$, \\ Yajun $\mathrm{Gu}^{4}$, Ting $\mathrm{Bei}^{4}$, Xiaochen $\mathrm{Zhao}^{4}$, Bei Zhang ${ }^{4}$, Yuezong Bai ${ }^{4}$, Atocha Romero ${ }^{5}$, Meng Xu-Welliver ${ }^{6}$, \\ Changli Wang ${ }^{1}$, Zhenfa Zhang ${ }^{1}$, Bin Zhang ${ }^{1}$
}

${ }^{1}$ Department of Lung Cancer, Tianjin Lung Cancer Center, National Clinical Research Center for Cancer, Key Laboratory of Cancer Prevention and Therapy, Tianjin's Clinical Research Center for Cancer, Tianjin Medical University Cancer Institute and Hospital, Tianjin, China; ${ }^{2}$ Department of Anesthesiology, National Clinical Research Center for Cancer, Key Laboratory of Cancer Prevention and Therapy, Tianjin's Clinical Research Center for Cancer, Tianjin Medical University Cancer Institute and Hospital, Tianjin, China; ${ }^{3}$ Department of Oncology, the First Hospital of Lanzhou University, Lanzhou, China; ${ }^{4}$ The Medical Department, 3D Medicines Inc., Shanghai, China; ${ }^{5}$ Medical Oncology Department, Hospital Universitario Puerta de Hierro-Majadahonda, Madrid, Spain; ${ }^{6}$ Department of Radiation Oncology, The Ohio State University Wexner Medical Center, Columbus, OH, USA

Contributions: (I) Conception and design: D Yue, B Zhang, C Wang, Z Zhang; (II) Administrative support: D Yue, B Zhang, C Wang, Z Zhang; (III) Provision of study materials or patients: D Yue, B Zhang, W Liu, C Chen, Y Ma; (IV) Collection and assembly of data: D Yue, B Zhang, W Liu, C Chen, Y Ma; (V) Data analysis and interpretation: D Yue, B Zhang, W Liu, Z Zhang, C Wang, T Zhang, C Chen, L Cui, Y Gu, X Zhao, B Zhang, T Bei, A Romero, M Xu-Welliver; (VI) Manuscript writing: All authors; (VII) Final approval of manuscript: All authors.

\#These authors contributed equally to this work.

Correspondence to: Bin Zhang; Zhenfa Zhang; Changli Wang. Department of Lung Cancer, Tianjin Lung Cancer Center, National Clinical Research Center for Cancer, Key Laboratory of Cancer Prevention and Therapy, Tianjin's Clinical Research Center for Cancer, Tianjin Medical University Cancer Institute and Hospital, Huan Hu West Road, Tianjin 300060, China. Email: zhangbin_09@tmu.edu.cn; zhangzhenfa1973@163.com; wangchangli@tjmuch.com.

Background: There is currently a lack of effective biomarkers to evaluate efficacy of neoadjuvant therapy (NAT) for resectable non-small cell lung cancer (NSCLC) patients. Circulating tumor DNA (ctDNA) has been investigated as a non-invasive tool for the assessment of tumor burden and minimal residual disease (MRD). The utility of ctDNA profiling in reflecting NAT efficacy, however, has not been confirmed. This study explored the association of ctDNA change with treatment response to NAT and recurrence-free survival (RFS) after surgery.

Methods: Eligible patients with stage IB-IIIA NSCLC were retrospectively included if they had received neoadjuvant immunotherapy combined with chemotherapy (IO+Chemo), dual immunotherapy (IO+IO), or chemotherapy alone (Chemo). We conducted ctDNA profiling before and after NAT, after surgery, and during follow-ups using an ultra-deep lung cancer-specific MRD (LC-MRD) sequencing panel.

Results: A total of 22 patients who received NAT followed by surgery between August 2018 and July 2019 were included in this study. The major pathological response (MPR) rates were $58.33 \%(7 / 12)$ in the IO+Chemo group, $25.00 \%(1 / 4)$ in the IO+IO group, and $16.67 \%(1 / 6)$ in the Chemo group. The ctDNA dynamics during NAT were highly concordant with pathologic response, demonstrating 100\% sensitivity and $83.33 \%$ specificity, for an overall accuracy of $91.67 \%$. Pre-surgery detectable ctDNA (after NAT) trended to correlate with inferior RFS [hazard ratio (HR), 7.41; 95\% confidence interval (CI): 0.91-60.22, log-rank $\mathrm{P}=0.03]$. At 3-8 days after surgery, ctDNA was detectable in $31.8 \%$ of patients and was an independent risk factor for recurrence (HR, 5.37; 95\% CI: 1.27-22.67; log-rank $\mathrm{P}=0.01)$. The presence of ctDNA at 3 months after surgery showed $83 \%$ sensitivity and $90 \%$ specificity for predicting relapse (C-index, 0.79; 95\% CI: 0.62-0.95). During disease monitoring after surgery, molecular recurrence by means of ctDNA preceded radiographic relapse, with a median time of 6.83 months. 
Conclusions: This study investigated the potential of ctDNA in evaluating NAT efficacy in NSCLC, implying the high concordance between ctDNA and pathological response. We also set out the prognostic value of perioperative ctDNA in predicting recurrence.

Keywords: Non-small cell lung cancer (NSCLC); circulating tumor DNA (ctDNA); neoadjuvant therapy (NAT); recurrence

Submitted Nov 23, 2021. Accepted for publication Feb 21, 2022.

doi: $10.21037 /$ tlcr-22-106

View this article at: https://dx.doi.org/10.21037/tlcr-22-106

\section{Introduction}

Lung cancer is the leading cause of cancer death worldwide (1). For resectable non-small cell lung cancer (NSCLC) patients, radical surgery is the standard of care as recommended by the National Comprehensive Cancer Network (NCCN) guidelines. Yet, a considerable proportion of patients (ranging from $50 \%$ for IA disease to $80 \%$ for IIIA disease) eventually succumb to recurrence within 5 years after resection (2-4). Several trials have suggested that neoadjuvant and adjuvant systemic therapy and/or radiotherapy, collectively defined as perioperative therapy, can improve clinical outcomes with a benefit of $5 \%$ on overall survival (OS), based on which perioperative therapies are routinely applied $(3,5)$. Recent studies have revealed a promising efficacy and safety profile of neoadjuvant immunotherapy in NSCLC. In the phase 2 randomized NEOSTAR trial for operable NSCLC, the major pathological response (MPR) rates induced by nivolumab and nivolumab + ipilimumab were $24 \%(5 / 21)$ and $50 \%(8 / 16)$, respectively. Pathological complete response $(\mathrm{pCR})$ rate was higher in patients administrated dual immunotherapy $(\mathrm{IO}+\mathrm{IO})$ than in those treated with nivolumab alone (38\% vs. 10\%) (6). More recently, the NADIM clinical trial, which evaluated the combination of chemotherapy and immunotherapy has reported an unprecedented pCR of $63 \%$ (7) and 36-month progressionfree survival (PFS) and OS of $81.1 \%$ and $91.0 \%$, respectively, among patients with resectable, stage IIIA NSCLC (8) shifting the paradigm of stage III NSCLC from that of a lethal disease to a curable one. Similarly, in CheckMate 816, neoadjuvant nivolumab + chemotherapy increased pCR rate to $24 \%$ in NSCLC, higher than the $2.2 \%$ induced by chemotherapy alone (Chemo) (9). Currently, imaging modalities, such as computed tomography (CT) and positron emission tomography (PET), are utilized to evaluate response to treatment and detect postoperative relapse during surveillance. The discordance between radiologic and pathological response underpins the need to develop effective biomarkers that can accurately predict therapeutic efficacy $(10,11)$. In addition, the suboptimal detection limit of radiographic imaging makes minimal lesions unmeasurable when the long axis of the lesion is smaller than $10 \mathrm{~mm}$. Therefore, a biomarker that can detect molecular/minimal residual disease (MRD) prior to radiologic recurrence is also urgently needed to guide timely treatment when the tumor/cancer burden is minimal. Collectively, effective biomarkers that can evaluate treatment response and expedite the identification of patients destined to relapse are unmet needs during the perioperative period.

Tumor immune microenvironment (TIME) makers such as PD-L1 expression, abundance of tumor-infiltrating lymphocytes (TILs), CD $8^{+} \mathrm{T}$ cells, and $\mathrm{CD} 56^{\mathrm{dim}}$ natural killer cells, and density of M1/M2-tumor-associated macrophages (12-15) were reported to be associated with pathological response in primary tumors subjected to immunotherapy; however, testing TIME markers relies on tumor tissue samples, which needs an invasive access. Circulating tumor DNA (ctDNA), a component of cellfree DNA (cfDNA), can reflect inter-and intra-tumoral heterogeneity. The ctDNA could noninvasively track dynamics of tumor burden and has been proposed as a real-time biomarker that can provide both predictive and prognostic values. In several cancers, including lung cancer, bladder cancer, and colorectal cancer, ctDNAbased profiling following surgery has shown encouraging performance in revealing MRD (16-19). In addition, ctDNA clearance has been shown to pose correlations with better treatment efficacy in advanced cancers (20-23), based on which its' effects in evaluating the efficacy of neoadjuvant therapy (NAT) have also been exploited. In bladder cancers, ctDNA has been found to be correlated with response to NAT (24). As for lung cancer, no data have been revealed 
except for a few preliminary results of ongoing trials, including CheckMate 816 (NCT02998528) and NADIM trial (NCT03081689) released at the American Association for Cancer Research (AACR) annual meeting 2021 (9) and IASLC 2021 World Conference on Lung Cancer 2021, respectively (25). The former reported that ctDNA clearance was associated with pathologic response and the latter that pre-treatment ctDNA levels as well as posttreatment ctDNA detection identified patients at high risk of progression and death and outperformed radiological response assessed according to RECIST criteria $\mathrm{v} 1.1$ in the prediction of survival. Our study primarily aimed to evaluate the utility of ctDNA profiling using next-generation sequencing (NGS)-based lung cancer-specific MRD (LCMRD) panel to assess response to NAT. The association of pre-surgery and post-surgery ctDNA with postoperative relapse was also explored. We present the following article in accordance with the STARD reporting checklist (available at https://tlcr.amegroups.com/article/view/10.21037/tlcr$22-106 / \mathrm{rc})$.

\section{Methods}

\section{Participants and study design}

This was a cohort study investigating the feasibility of ctDNA to reflect treatment response and predict recurrence in surgical NSCLC patients. Patients with NSCLC who received neoadjuvant immunotherapy combined with chemotherapy (IO+Chemo), IO+IO, or Chemo between August 2018 and July 2019 at the Tianjin Medical University Cancer Institute and Hospital were retrospectively analyzed. Patients were included if they had a stage IB-IIIA resectable NSCLC, at least 1 radiologically measurable target lesion, and an Eastern Cooperative Oncology Group (ECOG) performance status (PS) of 0-1. Patients were excluded if they had distant organ metastasis, secondary primary tumors, EGFR, $A L K$, and other actionable driver mutations, previous exposure to anti-cancer therapy, including chemotherapy, radiotherapy, and immunotherapy, and previous exposure to immunosuppressive drugs within 3 weeks before NAT. The study protocol, standard operating procedure (SOP) of data collection, and case report form (CRF) were prospectively designed before the initiation of the study to guarantee the quality of data. Blood samples were procured from patients at 3-8 days before NAT, within 1 week before surgery, at 3-8 days after surgery, and every 2-3 months thereafter until recurrence. All plasma samples were compared against their paired white blood cell (WBC) control and subjected to ultra-deep sequencing with LC-MRD panel at 3D Medicines, Inc., a College of American Pathologists (CAP)-accredited and Clinical Laboratory Improvement Amendments (CLIA)-certified laboratory. Parallel CT surveillance was conducted to monitor disease. Patients who had at least 2 postoperative follow-ups were included. Patients were identified according to their electronic medical records. All procedures performed involving human participants were conducted in accordance with Declaration of Helsinki (as revised in 2013). This study was approved by the ethics committee of Tianjin Medical University Cancer Institute and Hospital (No. E2020444A), and written informed consents were provided by all the patients.

\section{Outcomes and assessment}

This study aimed to investigate the utility of ctDNA profiling using the LC-MRD panel to evaluate the therapeutic efficacy and predict recurrence. Radiologic imaging was performed on primary tumor lesions using CT scans before, after NAT, and at follow-ups postoperatively as per Response Evaluation Criteria in Solid Tumors version (RECIST) 1.1 (26). Recurrence-free survival (RFS) was defined as the time between the date of surgery and the date when local recurrence or distant relapses were diagnosed. ORR was defined as the frequency of patients who have had achieved complete response (CR) or partial response (PR) at two consecutive CT assessment at least 4 weeks apart. Hematoxylin and eosin (H\&E) staining of surgical resection was applied for assessing histopathologic responses. The definition of MPR involved showing no more than $10 \%$ residual viable tumor cells, while a pCR referred to no residual tumor cells. All the radiologic and pathological data were reviewed by at least 2 independent radiologists or pathologists. The data were last edited on 16 April 2021.

\section{LC-MRD-panel development}

The MRD was detected by an NGS panel that identifies somatic single nucleotide variants (SNVs), insertiondeletions, and fusions in 194 genes (Table S1, Appendix 1: Supplementary Methods), of which at least 1 mutation would occur in $>90 \%$ of NSCLC tumors. To build the panel, we first included exons covering recurrent mutations in driver genes of NSCLC from The Catalogue of Somatic Mutations in Cancer (COSMIC; https://cancer.sanger. 
ac.uk/cosmic). Next, we developed an iterative algorithm to maximize the coverage of patients with minimum panel size using sequencing data of 1,577 NSCLC samples in the 3DMED database (https://www.3dmedcare.com/ technology/data.htm). Mutations with top recurrence index were selected into the design. Last, we added important actionable mutations and Chinese NSCLC patient-specific mutations into the panel, with the final size of $153 \mathrm{~kb}$.

\section{LC-MRD-panel technical validation}

For determination of SNV/Indel limit of detection (LoD) of the LC-MRD ctDNA assay, ctDNA reference standards of known mutations (including EGFR p.L858R, EGFR p.E746_ A750del) were diluted with wild-type cfDNA reference standard to create variant allele frequency (VAF) titration series, including $0.3 \%, 0.2 \%, 0.1 \%$, and $0.05 \%$. As for the determination of fusion LoD, genomic DNA of the tumor cell line NCI-H2228 with fusion of ALK-EML4 was diluted in the cell line without ALK-EML4 fusion to generate the titration series of $1 \%, 0.5 \%, 0.25 \%$, and $0.125 \%$, with at least 3 replications for each titration point. These DNA pools were subsequently analyzed by the LC-MRD panel sequencing at the coverage of $30,000 \times$ and detected by digital droplet polymerase chain reaction (ddPCR) as reference. The LoD was defined as the lowest VAF at which $95 \%$ of replicates are reliably detected for the variant type. Accordingly, the LoD of SNV/Indel and fusion were determined to be $0.1 \%$ and $0.25 \%$, respectively (Figure S1).

Intra-assay precision was assessed for repeatability. We prepared 4 cfDNA mixture pools with known VAF of $2 \%, 1 \%, 0.5 \%$, and $0.2 \%$ for each variant using ctDNA reference standards or variant overexpressing cell lines and ran them in triplicate under the same operating conditions. Every 3 replications formed a combination, and the rate of consistent detection of a known mutation in each combination was calculated for repeatability. Interassay precision was evaluated for reproducibility. The same sample pools in the repeatability study were prepared and subjected to a subsequent run. Every 2 different batch replications formed a combination, and the rate of consistent detection in each combination was calculated for reproducibility. The repeatability and reproducibility of the LC-MRD panel assay turned out to be $100 \%$ (Table S2).

\section{Blood collection and processing}

We collected $20 \mathrm{~mL}$ blood samples in a Streck tube from each patient and centrifuged them at $1,600 \mathrm{~g}$ at $4{ }^{\circ} \mathrm{C}$ for 10 min within $2 \mathrm{~h}$ of collection. The plasma supernatant was collected and transferred to a new microfuge tube, followed by centrifugation at $16,000 \mathrm{~g}$ at $4{ }^{\circ} \mathrm{C}$ for $10 \mathrm{~min}$. The supernatant and cell pellets were collected separately and stored at $-80^{\circ} \mathrm{C}$ until DNA extraction.

\section{NGS and sequencing data analysis}

The NGS and sequencing data analyses were performed at 3D Medicines, Inc. $(27,28)$. Briefly, cfDNA and genomic DNA from the paired WBCs were extracted using QiAmp Circulating Nucleic Acid Kit (QIAGEN, Hilden, Germany) and QIAamp DNA Mini Kit (QIAGEN), respectively. DNA extracts (30-200 ng) were sheared to $250 \mathrm{bp}$ fragments using an S220 focused-ultrasonicator (Covaris, Woodbrun, MA, USA). The median cfDNA input was $47.13 \mathrm{ng}$ (range, 14.98 to $60 \mathrm{ng}$ ). The cfDNA and genomic DNA libraries were constructed using the $x$ Gen Prism DNA Library Prep Kit (Integrated DNA Technologies, Inc., Coralville, IA, USA). They were individually barcoded with unique molecular identifiers (UMI), followed by probe-based hybridization. The captured libraries were subsequently loaded onto a NovaSeq 6000 platform (Illumina, San Diego, CA, USA) for paired-end sequencing with a mean sequencing depth of 50,000x. Sequencing data were mapped to the human genome hg19. Single-strand and duplex consensus sequences were generated to remove errors yielded from sequencing and PCR. Background noise was further polished by a self-developed database with cell-free DNA sequencing data from a healthy Chinese population. Germline and clonal hematopoiesis-derived mutations were filtered out based on data obtained by NGS profiling of paired WBCs. An in-house model for detecting loci-specific variants was applied to improve specificity $(27,28)$.

\section{Statistical analysis}

Categorical variables were presented as absolute and relative frequencies, and numerical variables were denoted as mean \pm standard deviation (SD), median, and range. The pCR, MPR, and ctDNA response rates were compared using the Fisher's exact test. To evaluate the effects of ctDNA change on predicting pathological response, relative delta mean variant allele fraction ( $\mathrm{R} \Delta$ mean $\mathrm{VAF}$ ) was calculated to depict dynamic changes of ctDNA upon NAT among pathological responders (MPR) and non-responders (nonMPR). Relative delta mean VAF $=$ (mean VAF on treatment 
- mean VAF at baseline)/mean VAF at baseline (29,30). A receiver operator characteristic (ROC) curve was generated to analyze the sensitivity and specificity of R $\Delta$ mean VAF predicting pathological response. The cutoff for defining responders was determined at -0.98 as the threshold where the maximum sum of specificity and sensitivity arrived. A 2 -sided Fisher's exact test was conducted to compare ctDNA change between pathologic responders and non-responders. As the $\mathrm{LoD}$ of the panel assay used was $0.25 \%$ for fusions and $0.1 \%$ for $\mathrm{SNV} /$ insertions and deletions (Indels), only variants of $\mathrm{AF}>0.3 \%$ detected at baseline were analyzed to increase the accuracy of prediction. A Kaplan-Meier curve was generated using a log-rank test to analyze RFS. Cox proportional hazards model was employed to compute hazard ratios (HR) and $95 \%$ confidence intervals (CI). A multivariate Cox regression model was established to identify independent risk factors for recurrence. The C-index (31) and landmark (32) analyses were performed to analyze the association of ctDNA with recurrence. The correlations between serum tumor biomarkers and pathological response to NAT were analyzed by using the bivariate linear regression model. The levels of tumor biomarkers during neoadjuvant treatment were recorded. A two sided $\mathrm{P}$ value less than 0.05 was considered statistically significant. Statistical analysis was performed using SPSS software (version 25).

\section{Results}

\section{Patient characteristics and baseline plasma sample analysis}

A total of 22 patients who received NAT and surgery were enrolled between 7 August 2018 and 26 July 2019 (Figure S2). The median age of the cohort $(\mathrm{n}=22)$ was 62.50 years (range, 53 to 72 years) and the majority of patients were male $(77.27 \%, 17 / 22)$. Approximately half of the patients had stage III disease, and the majority had an ECOG performance score of 0 (95.45\%, 21/22). Sixtyfour percent of patients were classified as lung squamous cell carcinomas (LUSC). A high percentage of the cohort had a smoking history $(81.82 \%, 18 / 22)$. In the neoadjuvant setting, 12 cases were treated with $\mathrm{IO}+$ Chemo, 4 with $\mathrm{IO}+\mathrm{IO}$, and 6 with Chemo. The clinicopathological characteristics of cases are summarized in Table 1. We performed NGS on baseline plasma and paired WBC samples, which identified 71 mutations from the entire cohort $(\mathrm{n}=22)$. A summary of mutation frequency is shown in Table S3. Baseline RBM10 mutation was associated with inferior RFS (data not shown). Until 22 January 2021, the median follow-up time after surgery was 17.67 months (range, 2.83 to 23.41 months).

\section{The correlation between ctDNA and the efficacy of NAT}

The pCR and MPR rates were $41.67 \%$ (5/12) and 58.33\% $(7 / 12)$ in the IO+Chemo group, $25 \%(1 / 4)$ and $25 \%(1 / 4)$ in the IO+IO group, and $0(0 / 6)$ and $16.67 \%(1 / 6)$ in the Chemo group, respectively. Surgical histopathology displayed a median tumor regression of $68 \%$ (range, $5 \%$ to $100 \%$ ). It was found that MPR occurred in 9 cases, which were therefore regarded as pathological responders $(40.9 \%, 9 / 22)$ (Figure 1A). The remaining 13 cases had a tumor regression of only $5-80 \%$ and were defined as nonresponders. According to CT assessment, 17 (77.2\%, 17/22) cases achieved CR or PR, and $5(22.7 \%, 5 / 22)$ had stable disease (SD) (Table 2) upon NAT. A total of 10 cases had discordant pathologic and radiologic response. According to CT imaging, 1 case had SD, but was turned out to have an MPR, and $9 \mathrm{CR} / \mathrm{PR}$ cases were found to be non-MPR, showing $88.89 \%$ sensitivity, $30.77 \%$ specificity, and an overall accuracy of $54.55 \%$. Serendipitously, ctDNA demonstrated better association with pathologic response compared with CT (Table 2). The ctDNA dynamics, defined as a relative delta mean VAF (R $\Delta$ mean VAF) upon NAT, yielded a sensitivity of $100 \%$ (95\% CI: $54.07 \%$ to $100 \%$ ), a specificity of $83.33 \%$ (95\% CI: $35.88 \%$ to $99.58 \%$ ), and an overall accuracy of $91.67 \%$ (95\% CI: $61.52 \%$ to $99.79 \%$ ) when the $\mathrm{R} \Delta$ mean VAF cutoff was set to -0.98 (Figure $1 B$, Table 2).

We also sought to analyze the routinely used tumor biomarkers and found that serum carbohydrate antigen 19-9 (CA19-9), carcinoembryonic antigen (CEA), or neuronspecific enolase (NSE) was not associated with pathologic response (Figure S3). These results were consistent with previous findings that the predictive or prognostic value of the currently used serum tumor protein biomarkers are limited $(33,34)$.

When cases were stratified based upon the treatment regimens, those who had received $\mathrm{IO}+\mathrm{Chemo}$ obtained a numerically better response than the cases treated with IO+IO or Chemo alone (pCRs: $42 \%$ vs. $25 \%$ vs. $0 \%$ ). Consistently, the IO+Chemo group had a numerically higher rate of ctDNA response than the other 2 groups (Figure S4). Patients in the $\mathrm{IO}+\mathrm{Chemo}$ group showed a significant better ORR than those in the Chemo alone according to CT assessment. These data suggest that ctDNA dynamics, defined as the relative R $\Delta$ mean VAF, had 
Table 1 Characteristics of patients with NSCLC at baseline

\begin{tabular}{|c|c|c|c|}
\hline Characteristic & All $(n=22)$ & Patients with MPR $(n=9)$ & Patients without MPR $(n=13)$ \\
\hline \multicolumn{4}{|l|}{ Sex, n (\%) } \\
\hline Male & $17(77.27)$ & $9(100.0)$ & $8(61.54)$ \\
\hline Female & $5(22.73)$ & $0(0)$ & $5(38.46)$ \\
\hline \multicolumn{4}{|l|}{ Age, years } \\
\hline Median (range) & $62.50(53.00-72.00)$ & $63.00(53.00-68.00)$ & $62.00(53.00-72.00)$ \\
\hline \multicolumn{4}{|l|}{ Stage, n (\%) } \\
\hline I & $5(22.73)$ & $333.33)$ & $2(15.38)$ \\
\hline ॥ & $4(18.18)$ & $1(11.11)$ & $3(23.08)$ \\
\hline $\mathrm{Sq}$ & $14(63.64)$ & $8(88.89)$ & $6(46.15)$ \\
\hline Non-sq & $8(36.36)$ & $1(11.11)$ & $7(53.85)$ \\
\hline \multicolumn{4}{|l|}{ NAT, n (\%) } \\
\hline $10+10$ & $4(18.18)$ & $1(11.11)$ & $3(23.08)$ \\
\hline IO+Chemo & $12(54.55)$ & $7(77.78)$ & $5(38.46)$ \\
\hline Chemo & $6(27.27)$ & $1(11.11)$ & $5(38.46)$ \\
\hline \multicolumn{4}{|l|}{ Smoking, n (\%) } \\
\hline Yes & $18(81.82)$ & $9(100.0)$ & $9(69.23)$ \\
\hline
\end{tabular}

NSCLC, non-small cell lung cancer; MPR, major pathological response, defined as $10 \%$ or less of viable tumor cells in the resected lesions after neoadjuvant therapy; SD, standard deviation; Sq, lung squamous cell carcinomas; NAT, neoadjuvant therapy; IO, immunotherapy; Chemo, chemotherapy; ECOG score, Eastern Cooperative Oncology Group performance score.

a better association with neoadjuvant efficacy.

\section{$C t D N A$ detection for identifying $M R D$ and relapse}

We then investigated the dynamic changes in somatic mutation profiles of blood samples from our cohorts at treatment milestones and various postoperative followup time points to understand their feasibility in reflecting tumor burden or MRD. A total of 19 cases $(86 \%, 19 / 22)$ had mutation detected from plasma samples at baseline, and the detection rate of ctDNA decreased to $59.1 \%$ and $31.8 \%$, respectively, after neoadjuvant treatment and at the first follow-up ( 3 to 8 days after surgery) (Figure 2).

Until 22 January 2021 , a total of 8 cases had radiologically confirmed recurrence, ranging from 2.53 to 12.66 months after surgery. In 5 of $8(62.5 \%)$ participants with CT-confirmed relapse, ctDNA was detectable in the blood sample at 3 to 8 days after surgery, suggesting the reliable reflection of MRD. All of the 8 cases that relapsed had ctDNA detected at any follow-up, occurring between 0.16 and 8.36 months after surgery (Figure $3 A$ ). In participants without detectable ctDNA at any time point post-operatively, the recurrence rate was $0 \%(0 / 8)$. Additionally, in the 8 cases with CT-confirmed relapse, the 
A

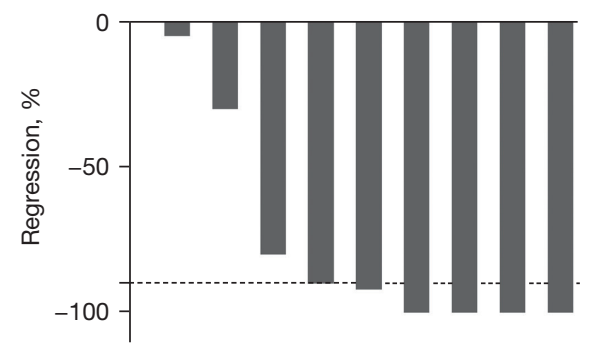

ctDNA+

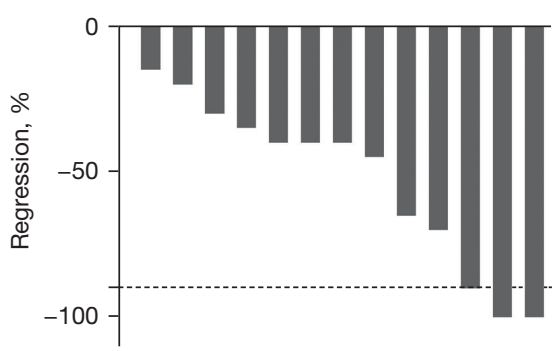

B

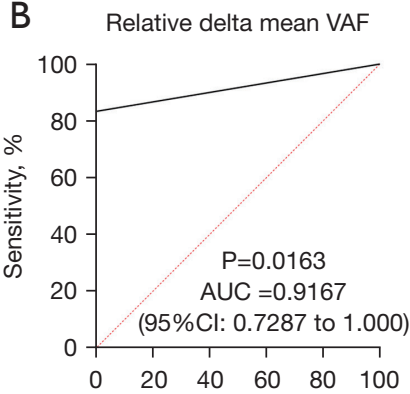

$100 \%$ - Specificity \%

Figure $1 \mathrm{ctDNA}$ and pathological response upon neoadjuvant therapy. (A) Pathologic response of patients with detectable or undetectable ctDNA upon neoadjuvant therapy. Blood samples were collected from patients after neoadjuvant therapy (within one week before surgery) and subjected to ctDNA profiling with LC-MRD panel. ctDNA + was defined as having any mutation detected from the panel used. The horizontal dashed line represents a major pathological response, defined as tumor regression of over $90 \%$. ctDNA- was defined as having no detectable mutation. (B) To evaluate the effects of ctDNA change on predicting pathological response, relative delta mean variant allele fraction (R $\Delta$ mean VAF) was calculated to depict dynamic changes of ctDNA upon neoadjuvant therapy among MPR and non-responders (non-MPR). Relative delta mean $\mathrm{VAF}=$ (mean VAF on treatment - mean VAF at baseline)/mean VAF at baseline. ROC curve showing the sensitivity and specificity of $\mathrm{R} \triangle$ mean VAF to predict pathological response. To aim for a maximum sum of specificity and sensitivity, the threshold for defining responders was determined to -0.98 . As the LoD of the panel assay used was $0.25 \%$ for fusions and $0.1 \%$ for SNV/insertions and deletions (Indels), only variants of $\mathrm{AF}>0.3 \%$ detected at baseline were analyzed to increase the accuracy of prediction. ctDNA, circulating tumor DNA; LC-MRD, lung cancer-specific minimal residual disease; MPR, major pathological response; ROC, receiver operator characteristic; VAF, variant allele frequency; LoD, limit of detection; SNV, single nucleotide variant; AUC, area under the curve.

Table 2 Concordance between ctDNA/radiologic and pathological response to neoadjuvant therapy in NSCLC

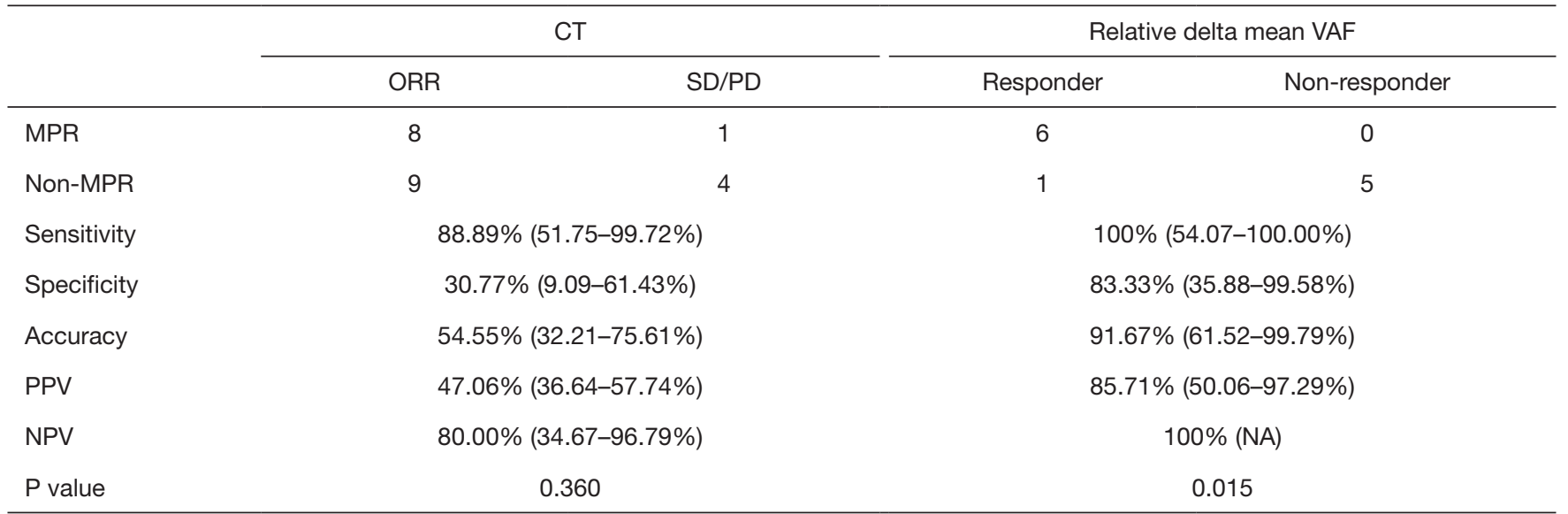

A 2-sided Fisher's exact test was conducted to analyze the association of pathologic response with ctDNA change and radiologic response. Tumor regression was assessed by CT imaging per RECIST v1.1. To aim for a maximum sum of specificity and sensitivity, the threshold for defining responders was determined to -0.98. ctDNA, circulating tumor DNA; NSCLC, non-small cell lung cancer; $\mathrm{CT}$, computed tomography; VAF, variant allele frequency; ORR, objective response rate; SD, stable disease; $\mathrm{PD}$, progressive disease; MPR, major pathologic response, defined as showing no more than $10 \%$ residual viable tumor cells; PPV, positive predictive value; NPV, negative predictive value; NA, not available. 


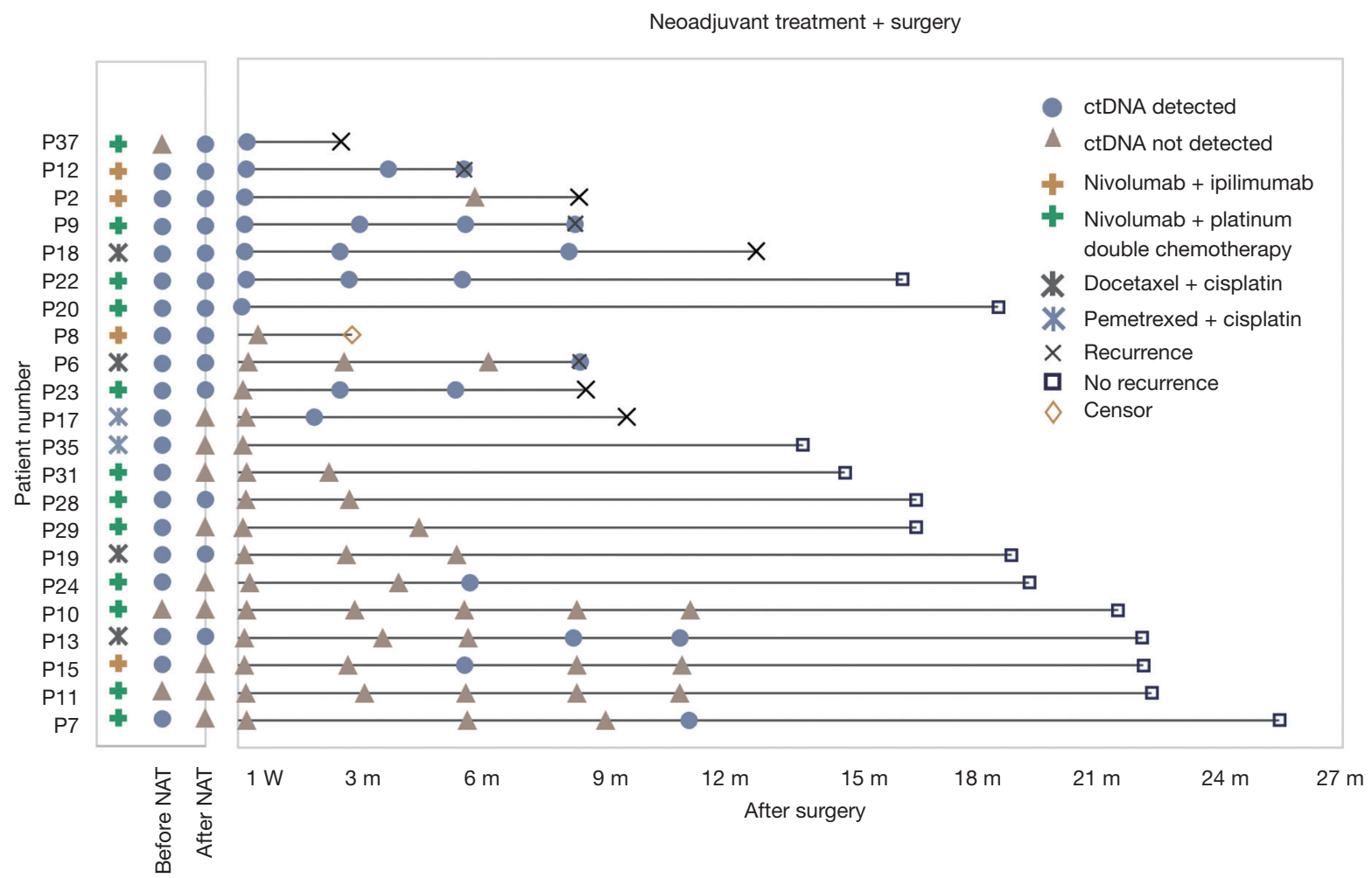

Figure 2 ctDNA monitoring at treatment milestones and during follow-ups. Blue circles denote the presence of ctDNA; while yellow triangles denote the absence of ctDNA. Neoadjuvant treatment regimens were represented by the indicated signs. Radiologic recurrence was denoted as multiplication sign. ctDNA, circulating tumor DNA.
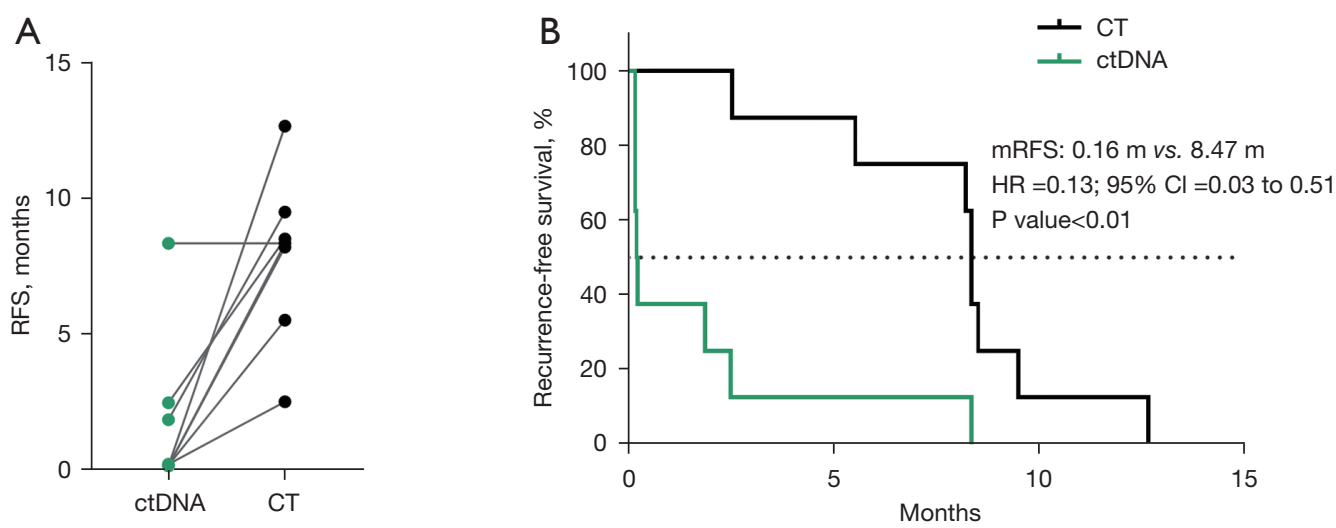

Figure 3 Monitoring of post-operative ctDNA predicts recurrence prior to radiologic imaging. (A) RFS determined by molecular or radiologic recurrence. The 8 patients with CT confirmed relapse were analyzed herein. Molecularly predicted recurrence was defined as the first presence of ctDNA at any time point after surgery. The presence of ctDNA was defined as having had any mutation detected from the used panel. Gray straight line denotes each patient's recurrence predicted by molecular profiling and radiologic imaging. (B) Kaplan-Meier survival curves of RFS determined by ctDNA profiling and CT imaging. Paired CT scan and ctDNA profiling were conducted at 3-8 days after surgery and every 2-3 months during the follow-up. ctDNA, circulating tumor DNA; RFS, recurrence-free survival; CT, computed tomography. 
A

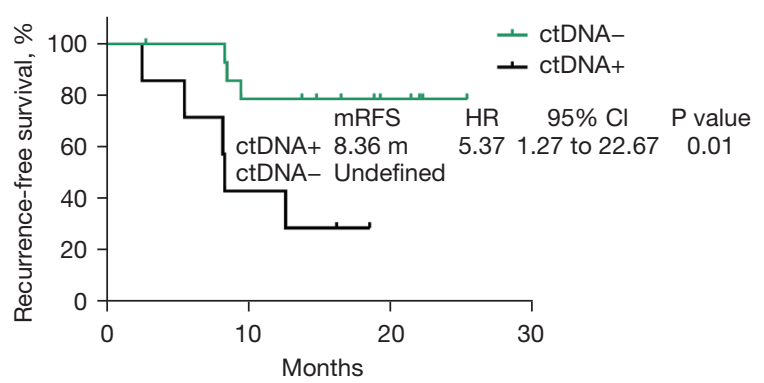

C

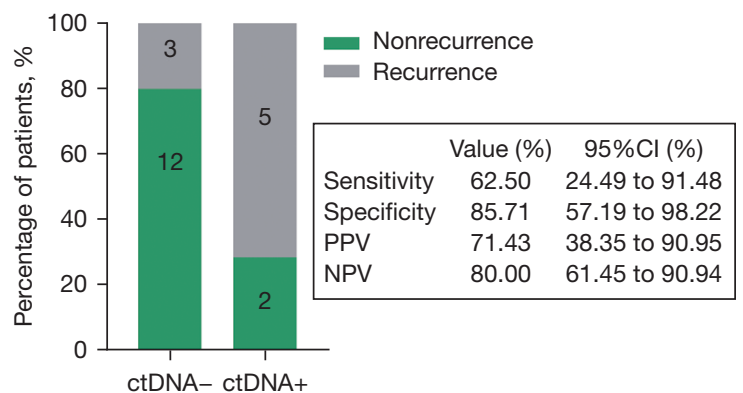

B

Three months after surgery

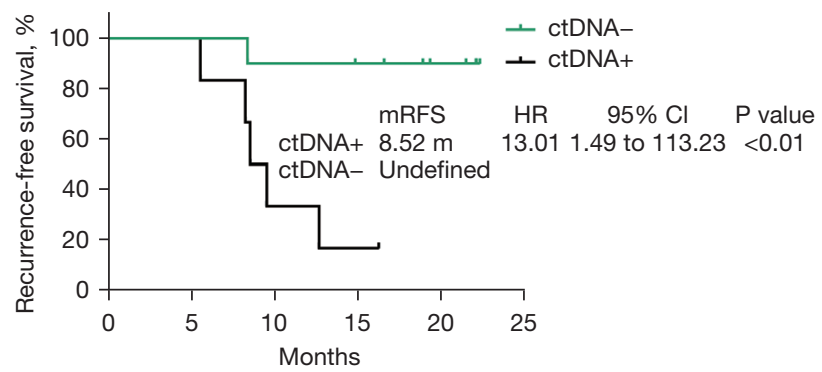

$\mathrm{D}$

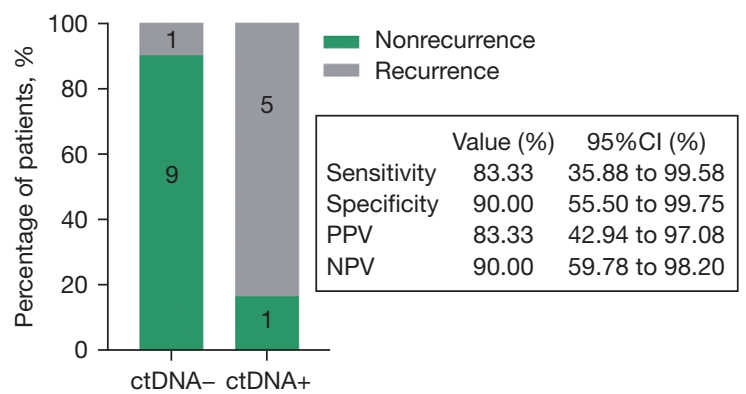

Figure 4 Absence of ctDNA after surgery correlates with better RFS. Kaplan-Meier survival curves depicting RFS of patients having had ctDNA detection at 1 week (n=22) (A) and 3 months $(n=16)$ (B) after surgery. The performance of ctDNA profiling at 1 week (C) and 3 months (D) postoperatively in predicting recurrence in reference to radiologic imaging. ctDNA was defined as any mutation detected from the used panel. Gray color indicates relapse cases and green color denotes relapse-free events confirmed by radiologic imaging. The characters in the columns represent the number of patients with the corresponding status. ctDNA, circulating tumor DNA; RFS, recurrence-free survival; mRFS, median RFS; PPV, positive predictive value; NPV, negative predictive value.

RFS indicated by ctDNA was significantly shorter than that by CT (Figure 3B) (ctDNA vs. CT, 0.16 vs. 8.47; logrank $\mathrm{P}<0.01)$. The ctDNA-determined recurrence was defined as the first detection of any mutation from the used panel after surgery. Post-surgery ctDNA presence preceded radiologic relapse with a median leading time of 6.83 months (0-12.50 months).

\section{Perioperative ctDNA detection for prognosis}

We also explored the prognostic and predictive value of ctDNA during the perioperative period to predict RFS in neoadjuvant immunotherapy and/or chemotherapytreated NSCLC. First, cases who had detectable ctDNA after NAT (at 1-8 days before surgery) trended towards an inferior RFS than those with no mutation detected (ctDNA+ vs. ctDNA-, 12.66 months vs. NA, HR, 7.41; $95 \%$ CI: 0.91 to 60.22 ; $\log$-rank $\mathrm{P}=0.03$, Figure S5). Postoperative ctDNA was found to be prognostic for
RFS. Patients who had detectable ctDNA at 3-8 days after surgery were associated with high risk for recurrence (ctDNA+ vs. ctDNA-, 8.36 months vs. NR; HR 5.37; 95\% CI: 1.27 to $22.67 ; \mathrm{P}=0.01$ ). Additionally, cases that had detectable ctDNA at 3 months postoperatively correlated with remarkably inferior RFS (ctDNA+ vs. ctDNA-, 8.52 months vs. NR; HR, 13.01; 95\% CI: 1.49 to 113.23 ; log-rank $\mathrm{P}<0.01$ ) (Figure 4). Orthogonal analysis of CTconfirmed relapse revealed the performance of postsurgery ctDNA in predicting recurrence. The ctDNA+ at 3-8 days after surgery showed $62.50 \%$ sensitivity, $85.71 \%$ specificity, $71.43 \%$ positive predictive value (PPV), and $80 \%$ negative predictive value (NPV) for identifying relapse, while ctDNA+ at 3 months after surgery displayed $83 \%$ sensitivity, $90 \%$ specificity, $83 \% \mathrm{PPV}$, and $90 \% \mathrm{NPV}$. Considering ctDNA as a time-varying covariate, we have also tried the C-index and landmark approaches to analyze the association of ctDNA with recurrence. Both the C-index or landmark analysis confirmed that ctDNA presence at 
one week [C-index, 0.72, 95\% CI: 0.57-0.87; landmark, 0-12 months, $\mathrm{P}=0.0101,12$ months later $(\mathrm{P}=0.0555)]$ and three months after surgery (C-index, 0.79, 95\% CI: $0.62-$ 0.95; landmark, 0-12 months: $\mathrm{P}=0.003,12$ months later $\mathrm{P}=0.0339$ ) was associated with recurrence. Of note, results obtained using these three approaches were consistent that ctDNA presence at three months after surgery was better associated with recurrence than ctDNA at one week.

We further looked into the predictive value of ctDNA after surgery within 3-8 days among patients treated with $\mathrm{IO}+$ Chemo. The ctDNA-negative participants showed a trend for longer RFS (ctDNA+ vs. ctDNA-, 13.4 months vs. $\mathrm{NR} ; \mathrm{P}=0.12$ ) than the ctDNA-positive cases. The predictive value of ctDNA at 3 months after surgery was of more interest, which showed that ctDNAnegative patients were more highly associated with better RFS than their counterparts (ctDNA+ vs. ctDNA-, 8.52 months vs. NR; log-rank $\mathrm{P}=0.03$ ), indicating post-surgery ctDNA was a prognostic biomarker for RFS in neoadjuvant immunotherapy-treated NSCLC (Figure S6).

Depending on data availability, univariable and multivariable analyses were conducted in participants who had ctDNA analyzed at 3 to 8 days after surgery to identify predictors of RFS. Smoking (HR 0.19; 95\% CI: 0.05 to $0.83 ; \log$-rank $\mathrm{P}=0.01)$ and ctDNA presence at 3 to 8 days after surgery (HR 5.37; 95\% CI: 1.27 to 22.67 ; logrank $\mathrm{P}=0.02)$ were significantly correlated with inferior RFS (Table S4). In the multivariate analysis, among smoking and ctDNA presence at 3 to 8 days after surgery, ctDNA presence was the only independent risk factor that associated with inferior RFS in neoadjuvant immunotherapy and/or chemotherapy-treated NSCLC (HR 4.59; 95\% CI: 1.04 to $20.33 ; \mathrm{P}=0.045$ ).

\section{Discussion}

Overall, our study revealed that lung cancer-specific ctDNA profiling during the perioperative period could evaluate efficacy of neoadjuvant immunotherapy and/ or chemotherapy, reflect post-surgery MRD, and predict recurrence in surgical NSCLC patients. Several findings are presented: (I) ctDNA change during NAT was highly concordant with pathologic response, showing $100 \%$ sensitivity and $83.33 \%$ specificity, for an overall accuracy of $91.67 \%$; (II) post-surgical ctDNA presence predicted recurrence, preceding radiographic relapse with a median leading time of 6.83 months; (III) both pre-surgery and post-surgery ctDNA predicted RFS; (IV) ctDNA presence at 3 months after surgery showed $83 \%$ sensitivity and $90 \%$ specificity for relapse prediction.

In the neoadjuvant setting, no effective biomarkers are available for evaluating therapeutic efficacy. Biomarkers reflecting treatment response may facilitate the pre-surgery identification of non-responders, thus for whom other optimal treatment regimens can be explored and offered. The CT imaging has been clinically utilized for response assessment but with limited concordance with pathologic response $(10,11)$. Change of ctDNA was shown to be predictive for treatment efficacy in advanced cancers (20-23). In locally advanced, unresectable, NSCLC ctDNA has been shown to predict survival outcomes (35). Similarly, its use to evaluate response to NAT has also been investigated. In this regard, ctDNA could predict pathological response of neoadjuvant chemoradiotherapy in patients with advanced rectal cancer (36). Similar results have been observed in bladder cancers (24). With respect to lung cancer, little is known about the effects of ctDNA changes on predicting NAT therapy. This study found that the ctDNA dynamics during NAT were highly associated with pathologic response (sensitivity, $100 \%$; specificity, $83 \%$ ). Perioperative ctDNA dynamics could predict neoadjuvant efficacy in NSCLC treated with neoadjuvant immunotherapy and/ or chemotherapy. Our finding agrees with the preliminary results of CheckMate 816 (NCT02998528) and NADIM trial (NCT03081689) presented at the AACR 2021 annual meeting (9) and IASLC 2021 World Conference on Lung Cancer 2021 (25), in which ctDNA clearance was reported to be associated with pathologic response and survival, respectively. Most recently, data of a single-arm trial (NCT02927301) investigating ctDNA association with pathologic response and outcomes were presented on the ESMO Immuno-Oncology Congress 2021, showing that ctDNA reductions post-atezolizumab correlated with pathologic response, and 2-year disease-free survival (DFS) was better in NSCLC patients who were ctDNA- postsurgery (37).

The optimal neoadjuvant regimen for resectable NSCLC patients remains unclear. A meta-analysis of 13 randomized control trials has shown that neoadjuvant chemotherapy significantly improved the OS of NSCLC patients compared with surgery alone (5). Moreover, immune checkpoint inhibitors (ICIs), which have demonstrated encouraging antitumor activities and few toxicities in advanced NSCLC patients, have attracted increasing attention in the perioperative setting. In a phase 2 trial, neoadjuvant nivolumab induced an MPR 
of $45 \%$, heralding an era of neoadjuvant immunotherapy for NSCLC patients (10). Finally, according to NADIM and CheckMate 816 data, it is becoming clear that the combination of chemotherapy and immunotherapy can significantly improve survival outcomes and multiple trials are therefore currently underway to confirm these findings (NCT02998528, NCT04941417, NCT04459611, NCT04197076, NCT03838159). Accordingly, in our study, the response elicited by nivolumab plus chemotherapy was numerically better than that by either the combinational nivolumab plus ipilimumab or single chemotherapeutic drug corresponding to the increase in pCR, MPR, or ctDNA clearance rates. Since the primary tumor can serve as an antigen source activating tumor-specific $T$ cells, we, therefore propose that immunotherapy may enhance chemotherapeutic efficacy, and the combinatory therapy might be a better neoadjuvant treatment option for resectable early-stage NSCLC patients (38-40). Given the small sample size and the retrospectively design, these data could not deliver a solid conclusion, and the analysis power was compromised. Further studies are required to confirm the results.

The presence of ctDNA after surgery could reflect the residual tumor burden and indicate recurrence prior to radiographic relapse (16-19). In our cohort, among patients without detectable ctDNA at any time point postoperatively, no recurrence occurred (0/8). All of the 8 cases that had CT-confirmed recurrence had detectable ctDNA at any follow-up after surgery, occurring between 0 and 12.50 months (median 6.83 months) ahead of radiologic recurrence. Consistently, despite the various definitions of ctDNA recurrence and different NGS panels employed, a leading time of ctDNA-determined relapse prior to radiologic relapse has been reported in multiple cancers, including lung cancer, bladder cancer, and colorectal cancers (16-19). That leading time in detecting molecular recurrence may open a window for earlier intervention. From our observation, patients who were ctDNA positive at 3 months after surgery exhibited a significantly inferior RFS than ctDNA negative cases, suggesting patients who are ctDNA-positive after surgery have a high risk of relapse and may need adjuvant therapy or maintenance therapy. This hypothesis was favored by the data revealed most recently in bladder cancers (41). Multiple ongoing trials exploring the ctDNA-determined MRD in lung cancer will reveal more data regarding its guiding value in the perioperative setting and during surveillance (ClinicalTrials. gov No. NCT04585477, NCT04385368, NCT04642469,
NCT04585490, NCT04367311, NCT04267237, NCT0425699, NCT04611776).

For ctDNA detection, our study employed a welldesigned LC-MRD ultra-deep sequencing panel, which was developed to maximize the coverage of patients with minimum panel size using sequencing data of 1,577 Chinese NSCLC cases in the 3DMed database. The presence of ctDNA at 3 months after surgery identified recurrence with $83 \%$ sensitivity and $90 \%$ specificity, comparable with the $92 \%$ and $90 \%$ in Signatera, and inferior to the $93 \%$ and 97\% in CAPP-Seq. Still, this panel has several advantages over the customized NGS assays. As reported, Signatera and CAPP-seq, which represent tumor-informed and tumor-uniformed strategy, respectively, were applied for ctDNA analysis in early-stage NSCLC $(16,19)$. Signatera was developed with a tumor-informed algorithm, aiming to detect patient-specific somatic variants; therefore, it was able to obtain a good accuracy with high sensitivity and specificity. Of note, the first evaluation time point of Signatera was at 30 days after surgery (16), which may not be sufficiently timely as a few patients were reported to have relapsed within 3 months postoperatively $(3,4,42)$. Similarly, in the CAPP-Seq study, the first time point of postoperative ctDNA detection was within 1 month after surgery; whereas in our observation, the presence of ctDNA within 3-8 days after surgery could also independently predict recurrence. Additionally, ctDNA based profiling can minimize the tumor heterogeneity. It is well known that clonal hematopoiesis-derived mutations constitute an important source of false positive rates (43). This study utilized an ultra-deep ctDNA sequencing with a paired WBC control, which could offset clonal evolution to some extent. Moreover, except for the variants selected by an iterative algorithm, this panel included other actionable mutations and Chinese NSCLC patient-specific mutations to improve clinical applicability, thus potentially facilitating the guiding of subsequent treatments.

This work was limited by its retrospective nature. The potential use of combinational agents during the operation and follow-ups may affect data interpretations. A small sample size and a lack of clinical validation are other significant limitations, for which the power of data was compromised to some extent, particularly in the subgroup analysis. Nevertheless, this study has shown that ctDNA dynamics upon NAT demonstrate high sensitivity and specificity for predicting NAT efficacy in resectable NSCLC patients. Monitoring of ctDNA presence and dynamic changes can be used as a predictor of benefit 
from neoadjuvant immunotherapy and/or chemotherapy in patients with NSCLC, which may potentially assist therapeutic decision-making and personalized treatment. Also, both pre-surgery and post-surgery ctDNA were found to be highly associated with RFS. Collectively, we envision the potential of ctDNA monitoring in guiding patients' management in the perioperative setting to define treatment response to NAT, identify MRD after surgery, and preemptively predict relapse. Prospective studies with larger sample sizes are warranted to validate our findings.

This is one of the first studies to reveal the potential of ctDNA in evaluating NAT efficacy in surgical NSCLC, demonstrating the high concordance between ctDNA and pathologic response. We also set out the prognostic value of perioperative ctDNA in predicting recurrence.

\section{Acknowledgments}

The authors appreciate the academic support from the AME Lung Cancer Collaborative Group. We would like to thank Hao Chen, Jie Wang, Shuli Song, and Yajuan Zhu for their help in ctDNA sequencing and data analysis.

Funding: This work was supported by National Natural Science Foundation of China (82173038 to Dongsheng Yue, 81772484 to Changli Wang).

\section{Footnote}

Reporting Checklist: The authors have completed the STARD reporting checklist. Available at https://tlcr.amegroups.com/ article/view/10.21037/tlcr-22-106/rc

Data Sharing Statement: Available at https://tlcr.amegroups. com/article/view/10.21037/tlcr-22-106/dss

Conflicts of Interest: All authors have completed the ICMJE uniform disclosure form (available at https://tlcr.amegroups. com/article/view/10.21037/tlcr-22-106/coif). LC, YG, $\mathrm{TB}, \mathrm{XZ}$, Bei Z, and YB declare that they are employees of 3D Medicines Inc. Atocha Romero reports personal fees from AstraZeneca, outside the submitted work. The other authors have no conflicts of interest to declare.

Ethical Statement: The authors are accountable for all aspects of the work in ensuring that questions related to the accuracy or integrity of any part of the work are appropriately investigated and resolved. All procedures performed involving human participants were conducted in accordance with Declaration of Helsinki (as revised in 2013). This study was approved by the Ethics committee of Tianjin Medical University Cancer Institute and Hospital (No. E2020444A). Written informed consent was provided by individual participants.

Open Access Statement: This is an Open Access article distributed in accordance with the Creative Commons Attribution-NonCommercial-NoDerivs 4.0 International License (CC BY-NC-ND 4.0), which permits the noncommercial replication and distribution of the article with the strict proviso that no changes or edits are made and the original work is properly cited (including links to both the formal publication through the relevant DOI and the license). See: https://creativecommons.org/licenses/by-nc-nd/4.0/.

\section{References}

1. Sung H, Ferlay J, Siegel RL, et al. Global Cancer Statistics 2020: GLOBOCAN Estimates of Incidence and Mortality Worldwide for 36 Cancers in 185 Countries. CA Cancer J Clin 2021;71:209-49.

2. NCCN Clinical Practice Guidelines in Oncology. NonSmall Cell Lung Cancer. Version 4. 2021 [database on the Internet]. National Comprehensive Cancer Network (NCCN). 2021. Available online: www.nccn.org. Accessed: June 10, 2021.

3. Pignon JP, Tribodet H, Scagliotti GV, et al. Lung adjuvant cisplatin evaluation: a pooled analysis by the LACE Collaborative Group. J Clin Oncol 2008;26:3552-9.

4. Goldstraw P, Crowley J, Chansky K, et al. The IASLC Lung Cancer Staging Project: proposals for the revision of the TNM stage groupings in the forthcoming (seventh) edition of the TNM Classification of malignant tumours. J Thorac Oncol 2007;2:706-14.

5. Song WA, Zhou NK, Wang W, et al. Survival benefit of neoadjuvant chemotherapy in non-small cell lung cancer: an updated meta-analysis of 13 randomized control trials. J Thorac Oncol 2010;5:510-6.

6. Cascone T, William WN Jr, Weissferdt A, et al. Neoadjuvant nivolumab or nivolumab plus ipilimumab in operable non-small cell lung cancer: the phase 2 randomized NEOSTAR trial. Nat Med 2021;27:504-14.

7. Provencio M, Nadal E, Insa A, et al. Neoadjuvant chemotherapy and nivolumab in resectable non-small-cell lung cancer (NADIM): an open-label, multicentre, singlearm, phase 2 trial. Lancet Oncol 2020;21:1413-22.

8. Provencio M, Nadal E, Insa A, et al. OA20.01 Long Term 
Survival in Operable Stage Iiia Nsclc Patients Treated With Neoadjuvant Nivolumab Plus Chemotherapy Nadim Study. J Thorac Oncol 2021;16:S883.

9. Forde PM, Spicer J, Lu S, et al. Abstract CT003: Nivolumab (NIVO) + platinum-doublet chemotherapy (chemo) vs chemo as neoadjuvant treatment (tx) for resectable (IB-IIIA) non-small cell lung cancer (NSCLC) in the phase 3 CheckMate 816 trial. Cancer Res 2021;81:CT003.

10. Forde PM, Chaft JE, Smith KN, et al. Neoadjuvant PD-1 Blockade in Resectable Lung Cancer. N Engl J Med 2018;378:1976-86.

11. William WN Jr, Pataer A, Kalhor N, et al. Computed tomography RECIST assessment of histopathologic response and prediction of survival in patients with resectable non-small-cell lung cancer after neoadjuvant chemotherapy. J Thorac Oncol 2013;8:222-8.

12. Riaz N, Havel JJ, Makarov V, et al. Tumor and Microenvironment Evolution during Immunotherapy with Nivolumab. Cell 2017;171:934-949.e16.

13. Wagner JA, Rosario M, Romee R, et al. CD56bright NK cells exhibit potent antitumor responses following IL-15 priming. J Clin Invest 2017;127:4042-58.

14. Li X, Liu R, Su X, et al. Harnessing tumor-associated macrophages as aids for cancer immunotherapy. Mol Cancer 2019;18:177.

15. Yang G, Su X, Yang H, et al. Neoadjuvant programmed death-1 blockade plus chemotherapy in locally advanced esophageal squamous cell carcinoma. Ann Transl Med 2021;9:1254

16. Abbosh C, Birkbak NJ, Wilson GA, et al. Phylogenetic ctDNA analysis depicts early-stage lung cancer evolution. Nature 2017;545:446-51.

17. Reinert T, Henriksen TV, Christensen E, et al. Analysis of Plasma Cell-Free DNA by Ultradeep Sequencing in Patients With Stages I to III Colorectal Cancer. JAMA Oncol 2019;5:1124-31.

18. Parikh AR, Van Seventer EE, Siravegna G, et al. Minimal Residual Disease Detection using a Plasma-only Circulating Tumor DNA Assay in Patients with Colorectal Cancer. Clin Cancer Res 2021;27:5586-94.

19. Chaudhuri AA, Chabon JJ, Lovejoy AF, et al. Early Detection of Molecular Residual Disease in Localized Lung Cancer by Circulating Tumor DNA Profiling. Cancer Discov 2017;7:1394-403.

20. Xia S, Ye J, Chen Y, et al. Parallel serial assessment of somatic mutation and methylation profile from circulating tumor DNA predicts treatment response and impending disease progression in osimertinib-treated lung adenocarcinoma patients. Transl Lung Cancer Res 2019;8:1016-28.

21. Jin Y, Chen DL, Wang F, et al. The predicting role of circulating tumor DNA landscape in gastric cancer patients treated with immune checkpoint inhibitors. Mol Cancer 2020;19:154.

22. Song Y, Hu C, Xie Z, et al. Circulating tumor DNA clearance predicts prognosis across treatment regimen in a large real-world longitudinally monitored advanced nonsmall cell lung cancer cohort. Transl Lung Cancer Res 2020;9:269-79.

23. Provencio M, Serna-Blasco R, Franco F, et al. Analysis of circulating tumour DNA to identify patients with epidermal growth factor receptor-positive non-small cell lung cancer who might benefit from sequential tyrosine kinase inhibitor treatment. Eur J Cancer 2021;149:61-72.

24. Powles T, Kockx M, Rodriguez-Vida A, et al. Clinical efficacy and biomarker analysis of neoadjuvant atezolizumab in operable urothelial carcinoma in the ABACUS trial. Nat Med 2019;25:1706-14.

25. Romero A, Nadal E, Serna R, et al. OA20.02 PreTreatment Levels of ctDNA for Long-Term Survival Prediction in Stage IIIA NSCLC Treated With Neoadjuvant Chemo-Immunotherapy. J Thorac Oncol 2021;16:S883-4.

26. Eisenhauer EA, Therasse P, Bogaerts J, et al. New response evaluation criteria in solid tumours: revised RECIST guideline (version 1.1). Eur J Cancer 2009;45:228-47.

27. von Wahlde MK, Timms KM, Chagpar A, et al. Intratumor Heterogeneity of Homologous Recombination Deficiency in Primary Breast Cancer. Clin Cancer Res 2017;23:1193-9.

28. Wang Z, Duan J, Cai S, et al. Assessment of Blood Tumor Mutational Burden as a Potential Biomarker for Immunotherapy in Patients With Non-Small Cell Lung Cancer With Use of a Next-Generation Sequencing Cancer Gene Panel. JAMA Oncol 2019;5:696-702.

29. Thompson JC, Carpenter EL, Silva BA, et al. Serial Monitoring of Circulating Tumor DNA by NextGeneration Gene Sequencing as a Biomarker of Response and Survival in Patients With Advanced NSCLC Receiving Pembrolizumab-Based Therapy. JCO Precis Oncol 2021;5:ePO.

30. Raja R, Kuziora M, Brohawn PZ, et al. Early Reduction in ctDNA Predicts Survival in Patients with Lung and Bladder Cancer Treated with Durvalumab. Clin Cancer Res 2018;24:6212-22. 
31. Romero A, García-Sáenz JA, Fuentes-Ferrer M, et al. Correlation between response to neoadjuvant chemotherapy and survival in locally advanced breast cancer patients. Ann Oncol 2013;24:655-61.

32. Rupji M, Zhang X, Kowalski J. CASAS: Cancer Survival Analysis Suite, a web based application. F1000Res 2017;6:919.

33. Chen KZ, Lou F, Yang F, et al. Circulating Tumor DNA Detection in Early-Stage Non-Small Cell Lung Cancer Patients by Targeted Sequencing. Sci Rep 2016;6:31985.

34. Ma S, Shen L, Qian N, et al. The prognostic values of CA125, CA19.9, NSE, AND SCC for stage I NSCLC are limited. Cancer Biomark 2011-2012;10:155-62.

35. Provencio M, Majem M, Guirado M, et al. Phase II clinical trial with metronomic oral vinorelbine and triweekly cisplatin as induction therapy, subsequently concomitant with radiotherapy (RT) in patients with locally advanced, unresectable, non-small cell lung cancer (NSCLC). Analysis of survival and value of ctDNA for patient selection. Lung Cancer 2021;153:25-34.

36. Wang Y, Yang L, Bao H, et al. Utility of ctDNA in predicting response to neoadjuvant chemoradiotherapy and prognosis assessment in locally advanced rectal cancer: A prospective cohort study. PLoS Med 2021;18:e1003741.

37. Kris MG, Grindheim JM, Chaft JE, et al. 10 Dynamic

Cite this article as: Yue D, Liu W, Chen C, Zhang T, Ma Y, Cui L, Gu Y, Bei T, Zhao X, Zhang B, Bai Y, Romero A, Xu-Welliver M, Wang C, Zhang Z, Zhang B. Circulating tumor DNA predicts neoadjuvant immunotherapy efficacy and recurrence-free survival in surgical non-small cell lung cancer patients. Transl Lung Cancer Res 2022;11(2):263-276. doi: 10.21037/tlcr-22-106 circulating tumour DNA (ctDNA) response to neoadjuvant (NA) atezolizumab (atezo) and surgery (surg) and association with outcomes in patients (pts) with NSCLC. Ann Oncol 2021;32:S1373.

38. Liu J, Blake SJ, Yong MC, et al. Improved Efficacy of Neoadjuvant Compared to Adjuvant Immunotherapy to Eradicate Metastatic Disease. Cancer Discov 2016;6:1382-99.

39. Salmon H, Idoyaga J, Rahman A, et al. Expansion and Activation of CD103(+) Dendritic Cell Progenitors at the Tumor Site Enhances Tumor Responses to Therapeutic PD-L1 and BRAF Inhibition. Immunity 2016;44:924-38.

40. Goldberg MV, Maris CH, Hipkiss EL, et al. Role of PD-1 and its ligand, B7-H1, in early fate decisions of CD8 T cells. Blood 2007;110:186-92.

41. Powles T, Assaf ZJ, Davarpanah N, et al. ctDNA guiding adjuvant immunotherapy in urothelial carcinoma. Nature 2021;595:432-7.

42. Consonni D, Pierobon M, Gail MH, et al. Lung cancer prognosis before and after recurrence in a populationbased setting. J Natl Cancer Inst 2015;107:djv059.

43. Ptashkin RN, Mandelker DL, Coombs CC, et al. Prevalence of Clonal Hematopoiesis Mutations in TumorOnly Clinical Genomic Profiling of Solid Tumors. JAMA Oncol 2018;4:1589-93. 


\section{Supplementary methods for LC_MRD panel design}

We developed a five-phase process to optimize genomic regions for the LC_MRD-panel. NSCLC driver genes recommended by the National Comprehensive Cancer Network (NCCN) guidelines (44), suspected NSCLC driver genes, and Chinesespecific genomic alternations in NSCLC were included in phases 1, 4, 5. In phases 2 and 3, an iterative method was used to maximize both the number of patients covered and SNVs per patient. The latter was determined by the "Recurrence Index" (RI), defined as the number of NSCLC patients harboring SNVs within a specific kilobase of exonic sequence (45). In phases 2 and 3, we analyzed non-silent SNVs identified in sequencing data of 1577 Chinese NSCLC tissue samples in the 3DMED database. Cutoffs for RI and patients per exon were chosen to enrich known or potential driver genes (45). The following steps were used to design the LC_MRD-panel.

Phase 1 (Known drivers)

Initial seed genes were chosen based upon their mutation frequency in NSCLC. COSMIC (https://cancer.sanger.ac.uk/ cosmic) was used to identify known driver genes that are recurrently mutated in $\geq 9 \%$ of NSCLC. Based on the previously documented SNV pattern, certain exons from these genes were chosen. Single exons from genes with recurrent mutations that occurred at low frequency but had good evidence for being driver mutations were also included in the seed list (45-47).

Phase 2 (Max. coverage)

For each exon with a top 20\% RI and a top 20\% SNV frequency ranking in the 1577 Chinese NSCLC, we selected the exon with the highest RI that identified at least 1 additional patient compared to the prior phase. Among exons with equally high RI, we included the exon with the least overlap among patients already captured by the selector. The process was repeated until no more exons satisfied the aforesaid criteria.

Phase 3 (Max. median)

For each remaining exon with a top $25 \% \mathrm{RI}$ and a top $25 \% \mathrm{SNV}$ frequency ranking in the 1577 Chinese NSCLC, we selected the exon that would result in the biggest rise in patients with at least two SNV. For exons that are equally good, the exon with the highest RI was chosen. Repeat was performed until no other exons met the above criteria.

Phase 4 (NCCN recommended NSCLC driver genes)

We included all exons from additional genes recommended by NCCN guidelines (44).

Phase 5 (Chinese-specific mutation)

We also included genomic alternations that identified in $>0.5 \%$ of internal Chinese NSCLC tissue sample, or mutations that were previously reported (Asia WES data) for more than five times. 
Table S1 Gene list of lung cancer (LC)-MRD panel

\begin{tabular}{|c|c|c|c|c|}
\hline$A B C B 11$ & CTR9 & HOXA11 & NOTCH1 & RFC3 \\
\hline ACVR1B & CUX1 & $I D H 1$ & NRAS & ROS1 \\
\hline$A C V R 2 A$ & $D D R 2$ & IGF1R & NRG3 & $R U N X 1 T 1$ \\
\hline AFF3 & $D D \times 10$ & $I K Z F 1$ & NSD2 & $R X R A$ \\
\hline AKT3 & ELF3 & $J A K 1$ & NTRK3 & $S F P Q$ \\
\hline$A L K$ & $E P 300$ & JAK3 & PALB2 & SH2B3 \\
\hline ANK1 & EPHA2 & $K D M 5 A$ & PARP1 & SLC25A13 \\
\hline$A R N T$ & ETV6 & КМТ2C & PHOX2B & SMAD3 \\
\hline ATM & $E Z R$ & KMT2D & PIКЗСА & SMAD4 \\
\hline ATP2B3 & FAM135B & KRAS & PIКЗСВ & SMARCA4 \\
\hline ATR & FANCA & LATS2 & PIKЗCD & SMO \\
\hline$B C L 11 B$ & FANCM & LIG1 & PIK3R1 & SOX9 \\
\hline$B R A F$ & FAT4 & $L R P 1 B$ & PIK3R2 & SPTA1 \\
\hline BRCA2 & FES & LZTR1 & PIK3R3 & STAT3 \\
\hline CACNA1D & FGF3 & $M A P 2 K 1$ & POLD1 & STK11 \\
\hline CCNO & FOXP1 & MGMT & PTEN & TGFBR1 \\
\hline $\mathrm{CDH} 10$ & FUS & MMS19 & PTPN11 & TGFBR2 \\
\hline $\mathrm{CDH} 11$ & GATA3 & MSH3 & PTPN13 & THBS2 \\
\hline$C D K 8$ & GLI1 & MSH4 & PTPRD & TIMELESS \\
\hline$C D K N 2 A$ & GLI3 & MSH6 & PTPRT & TP53 \\
\hline CHD2 & GNAQ & $M Y C$ & RAD50 & $U 2 A F 1$ \\
\hline CHD4 & GNAS & $M Y C N$ & $R A P 1 G D S 1$ & UGT1A1 \\
\hline CLTCL1 & GRIN2A & MYD88 & RASA1 & USP8 \\
\hline CNBP & HDAC2 & NCOR2 & $R B 1$ & $X A B 2$ \\
\hline CREB3L2 & $H G F$ & $N F 1$ & $R B M 10$ & XPO1 \\
\hline CREBBP & HIF1A & NFE2L2 & $R E C Q L$ & XRCC6 \\
\hline CTNNB1 & HLTF & NFKBIA & $R E T$ & ZBTB16 \\
\hline CTNND2 & HNF1A & $N K X 2-1$ & $R E V 1$ & \\
\hline
\end{tabular}



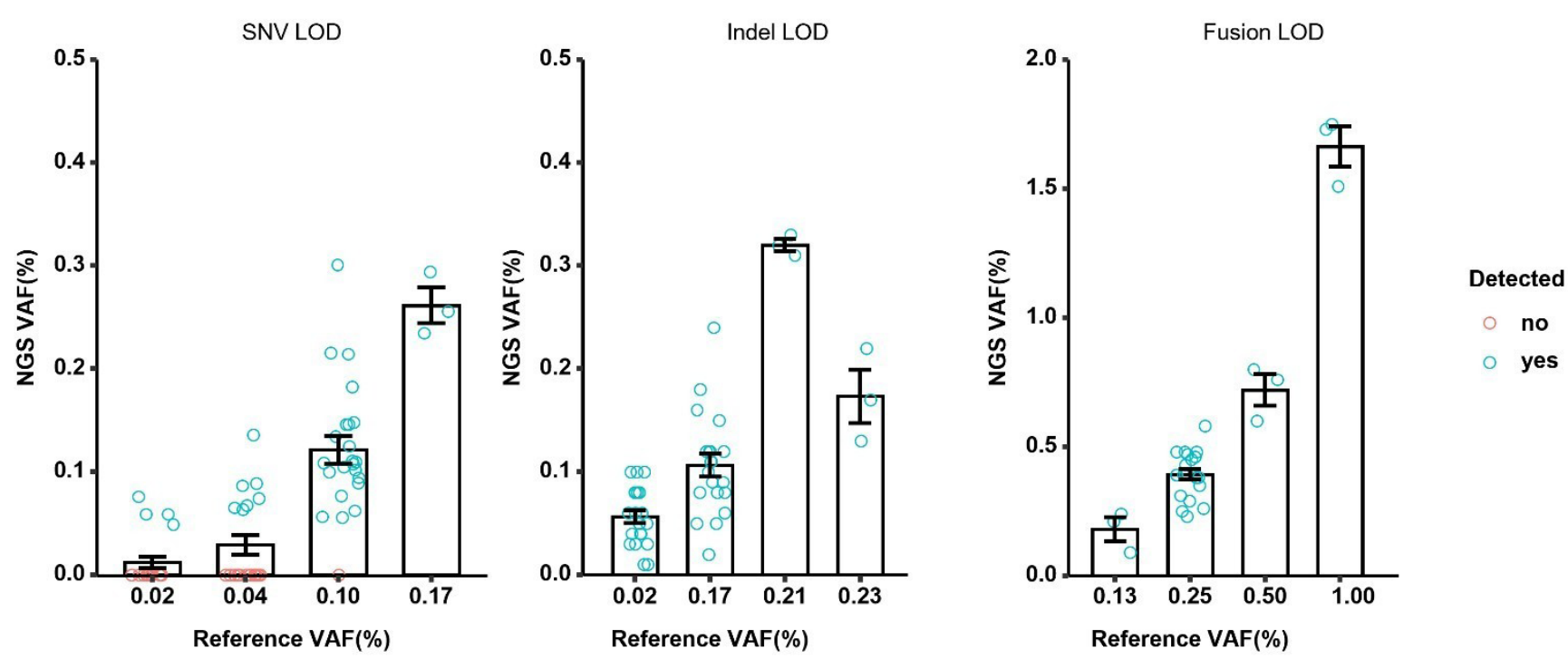

Figure S1 Limit of detection (LOD) determination for the LC-MRD assay. ctDNA reference standards of known mutations (EGFR p.L858R, EGFR p.E746_A750del, KRAS p.g12D and PIK3CA p.E545K) were diluted with wild-type ctDNA reference standard to create variant allele frequency (VAF) titration series, including $0.5 \%, 0.3 \%, 0.2 \%, 0.1 \%$, and $0.05 \%$. Genomic DNA of the tumor cell line NCI-H2228 with known ALK-EML4 rearrangement were diluted in cell line LS180 with no ALK-EML4 to generate VAF titration series of $1 \%, 0.5 \%, 0.25 \%, 0.125 \%, 0.05 \%$. All these DNA pools' VAF were detected by digital droplet PCR (ddPCR) as a golden standard. The limit of the detection was defined as the lowest VAF at which $95 \%$ of replicates are reliably detected for the variant type. LOD of SNV/ Indel and fusion were finally determined to be $0.1 \%$ and $0.25 \%$, respectively, at $30 \mathrm{ng}$ input. Values are presented as means \pm standard error of mean (SEM). LOD, limit of detection; SNV, single nucleotide variant, Indel, insertions and deletions; NGS, next-generation sequencing; $\mathrm{VAF}$, variant allele frequency. 
Table S2 Technical validation of LC-MRD panel

\begin{tabular}{|c|c|c|c|c|}
\hline $\begin{array}{l}\text { Gene \& amino acid sequence } \\
\text { change }\end{array}$ & Reference VAF & $\frac{\text { Repeatability }}{\text { batch } 1}$ & $\frac{\text { Repeatability }}{\text { batch } 2}$ & Reproducibility \\
\hline \multirow[t]{3}{*}{ PIK3CA_p.E545K } & $2.00 \%$ & $100 \%$ & $100 \%$ & $100 \%$ \\
\hline & $1.00 \%$ & $100 \%$ & $100 \%$ & $100 \%$ \\
\hline & $0.50 \%$ & $100 \%$ & $100 \%$ & $100 \%$ \\
\hline \multirow[t]{4}{*}{ EGFR_19DEL } & $2.00 \%$ & $100 \%$ & $100 \%$ & $100 \%$ \\
\hline & $1.00 \%$ & $100 \%$ & $100 \%$ & $100 \%$ \\
\hline & $0.50 \%$ & $100 \%$ & $100 \%$ & $100 \%$ \\
\hline & $0.20 \%$ & $100 \%$ & $100 \%$ & $100 \%$ \\
\hline \multirow{2}{*}{ KRAS_p.G12D } & $0.50 \%$ & $100 \%$ & $100 \%$ & $100 \%$ \\
\hline & $0.20 \%$ & $100 \%$ & $100 \%$ & $100 \%$ \\
\hline \multirow[t]{4}{*}{ EGFR_p.L858R } & $2.00 \%$ & $100 \%$ & $100 \%$ & $100 \%$ \\
\hline & $1.00 \%$ & $100 \%$ & $100 \%$ & $100 \%$ \\
\hline & $0.50 \%$ & $100 \%$ & $100 \%$ & $100 \%$ \\
\hline & $0.20 \%$ & $100 \%$ & $100 \%$ & $100 \%$ \\
\hline \multirow[t]{2}{*}{ ALK-EML4 fusion } & $2.00 \%$ & $100 \%$ & $100 \%$ & $100 \%$ \\
\hline & $1.00 \%$ & $100 \%$ & $100 \%$ & $100 \%$ \\
\hline
\end{tabular}

VAF, variant allele frequency. $19 \mathrm{DEL}$, deletion in exon 19. 


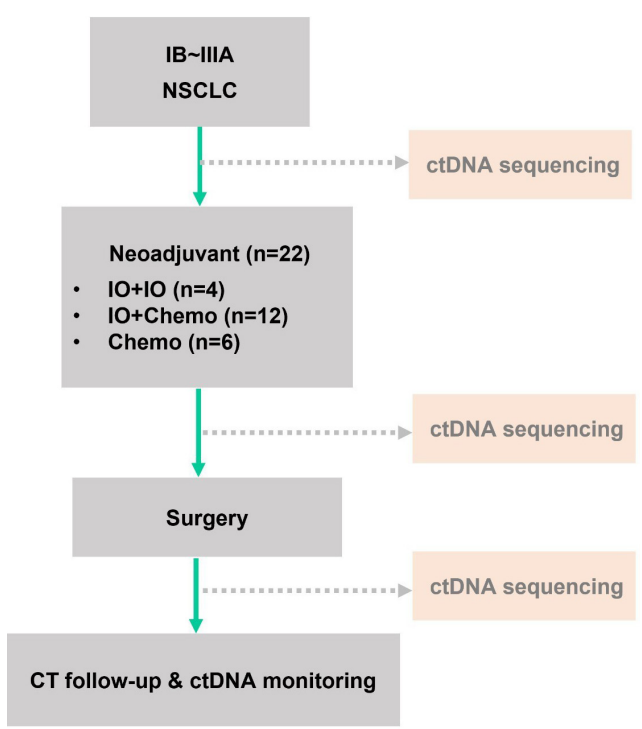

Figure S2 Schematic study design assessing the utility of ctDNA monitoring in early-stage resectable NSCLC. NSCLC, non-small cell lung cancer; IO, immunotherapy; Chemo, chemotherapy; CT, computed tomography; ctDNA, circulating tumor DNA. 


\begin{tabular}{|c|c|c|c|c|c|c|}
\hline Patient ID & $\begin{array}{l}\text { Plasma used for } \\
\text { library }(\mathrm{mL})\end{array}$ & $\begin{array}{l}\text { Mean depth } \\
\text { sequenced }\end{array}$ & $\begin{array}{l}\text { Somatic variants (protein } \\
\text { position) }\end{array}$ & Occurring region & Exonic function type & Allele fraction \\
\hline P2 & 11 & 9611.53 & EGFR_p.R831C & exonic & nonsynonymous SNV & 0.001438 \\
\hline P3 & 6 & 10080.7 & NFE2L2_p.D21H & exonic & nonsynonymous SNV & 0.001074 \\
\hline P3 & 6 & 10080.7 & ATR_p.W2379L & exonic & nonsynonymous SNV & 0.00125 \\
\hline P3 & 6 & 10080.7 & CTNNB1_p.L385I & exonic & nonsynonymous SNV & 0.000963 \\
\hline P6 & 6.5 & 7905.32 & TP53_p.E294* & exonic & stopgain & 0.02344 \\
\hline P6 & 6.5 & 7905.32 & TP53_p.C242F & exonic & nonsynonymous SNV & 0.027554 \\
\hline P6 & 6.5 & 7905.32 & CUX1_p.D1385N & exonic & nonsynonymous SNV & 0.002152 \\
\hline P6 & 6.5 & 7905.32 & EGFR_p.R831C & exonic & nonsynonymous SNV & 0.001079 \\
\hline P6 & 6.5 & 7905.32 & CDKN2A_p.D84N & exonic & nonsynonymous SNV & 0.029271 \\
\hline P7 & 2.5 & 7662.22 & EGFR_p.W817R & exonic & nonsynonymous SNV & 0.003755 \\
\hline P7 & 2.5 & 7662.22 & EGFR_p.L858R & exonic & nonsynonymous SNV & 0.000631 \\
\hline P8 & 7 & 4921.5 & HOXA11_p.G149D & exonic & nonsynonymous SNV & 0.00149 \\
\hline P8 & 7 & 4921.5 & EGFR_p.L858R & exonic & nonsynonymous SNV & 0.000885 \\
\hline P9 & 3 & 9029.69 & TP53_splicing & splicing & NA & 0.001179 \\
\hline P9 & 3 & 9029.69 & RBM10_p.Q440H & exonic & nonsynonymous SNV & 0.002657 \\
\hline P12 & 5 & 11787.8 & TP53_p.R249M & exonic & nonsynonymous SNV & 0.022144 \\
\hline P12 & 5 & 11787.8 & FAM135B_p.T1263T & exonic & synonymous SNV & 0.013483 \\
\hline P12 & 5 & 11787.8 & RUNX1T1_p.P235T & exonic & nonsynonymous SNV & 0.022403 \\
\hline P12 & 5 & 11787.8 & CDKN2A_p.R80* & exonic & stopgain & 0.019832 \\
\hline P12 & 5 & 11787.8 & RBM10_p.R744P & exonic & nonsynonymous SNV & 0.012148 \\
\hline P13 & 5.5 & 11261.5 & TP53_p.P301Qfs44 & exonic & frameshift deletion & 0.007208 \\
\hline P13 & 5.5 & 11261.5 & MBD4_p.D261G & exonic & nonsynonymous SNV & 0.004162 \\
\hline P13 & 5.5 & 11261.5 & CDKN2A_p.M54_G55insl & exonic & nonframeshift insertion & 0.00247 \\
\hline P14 & 6 & 12032 & TP53_splicing & exonic;splicing & frameshift deletion & 0.114985 \\
\hline P14 & 6 & 12032 & CTNND2_p.1420V & exonic & nonsynonymous SNV & 0.00531 \\
\hline P14 & 6 & 12032 & FAM135B_p.E544K & exonic & nonsynonymous SNV & 0.067722 \\
\hline P14 & 6 & 12032 & CDKN2A_p.V59E & exonic & nonsynonymous SNV & 0.001306 \\
\hline P15 & 6.5 & 11338.9 & KMT2D_p.P584L & exonic & nonsynonymous SNV & 0.001959 \\
\hline P15 & 6.5 & 11338.9 & TP53_p.E349* & exonic & stopgain & 0.001054 \\
\hline P15 & 6.5 & 11338.9 & TP53_p.R282W & exonic & nonsynonymous SNV & 0.003833 \\
\hline P17 & 6 & 7549.83 & TP53_splicing & splicing & NA & 0.001072 \\
\hline P17 & 6 & 7549.83 & SLC34A2_p.N495del & exonic & nonframeshift deletion & 0.000926 \\
\hline P17 & 6 & 7549.83 & EGFR_p.V441I & exonic & nonsynonymous SNV & 0.002403 \\
\hline P18 & 4 & 8715.18 & KRAS_p.G12E & exonic & nonframeshift substitution & 0.000788 \\
\hline P18 & 4 & 8715.18 & RUNX1T1_p.A461T & exonic & nonsynonymous SNV & 0.001217 \\
\hline P19 & 5.5 & 13730.7 & TP53_p.P250L & exonic & nonsynonymous SNV & 0.055791 \\
\hline P19 & 5.5 & 13730.7 & AFF3_p.R846C & exonic & nonsynonymous SNV & 0.001128 \\
\hline P19 & 5.5 & 13730.7 & CTNND2_p.A803D & exonic & nonsynonymous SNV & 0.016422 \\
\hline P19 & 5.5 & 13730.7 & FAM135B_p.Q814P & exonic & nonsynonymous SNV & 0.02377 \\
\hline P19 & 5.5 & 13730.7 & FAM135B_p.D443N & exonic & nonsynonymous SNV & 0.002147 \\
\hline P19 & 5.5 & 13730.7 & AR_p.S432F & exonic & nonsynonymous SNV & 0.016431 \\
\hline P20 & 5 & 11069 & TP53_p.G245C & exonic & nonsynonymous SNV & 0.415345 \\
\hline P20 & 5 & 11069 & NOTCH1_p.S2499Tfs*90 & exonic & frameshift deletion & 0.001017 \\
\hline P22 & 6 & 14973.9 & TP53_p.R282G & exonic & nonsynonymous SNV & 0.008348 \\
\hline P23 & 6.5 & 11881.5 & KMT2D_p.E856Q & exonic & nonsynonymous SNV & 0.315208 \\
\hline P23 & 6.5 & 11881.5 & KMT2D_p.E748Q & exonic & nonsynonymous SNV & 0.31836 \\
\hline P23 & 6.5 & 11881.5 & KMT2D_p.E649K & exonic & nonsynonymous SNV & 0.250229 \\
\hline P23 & 6.5 & 11881.5 & KMT2D_p.E631K & exonic & nonsynonymous SNV & 0.279321 \\
\hline P23 & 6.5 & 11881.5 & BCL11B_p.P268L & exonic & nonsynonymous SNV & 0.001996 \\
\hline P23 & 6.5 & 11881.5 & TP53_p.E336* & exonic & stopgain & 0.40132 \\
\hline P23 & 6.5 & 11881.5 & PIK3CA_p.E545K & exonic & nonsynonymous SNV & 0.009592 \\
\hline P23 & 6.5 & 11881.5 & PIK3CA_p.H1047R & exonic & nonsynonymous SNV & 0.004909 \\
\hline P23 & 6.5 & 11881.5 & SETD2_p.F1606L & exonic & nonsynonymous SNV & 0.004218 \\
\hline P23 & 6.5 & 11881.5 & NOTCH1_p.G1753Cfs*42 & exonic & frameshift deletion & 0.015579 \\
\hline P24 & 5 & 7722.51 & TP53_p.P278L & exonic & nonsynonymous SNV & 0.01941 \\
\hline P24 & 5 & 7722.51 & CDKN2A_p.W110* & exonic & stopgain & 0.011224 \\
\hline P28 & 4 & 8380.35 & TP53_p.P316Sfs*21 & exonic & frameshift insertion & 0.005092 \\
\hline P28 & 4 & 8380.35 & TP53_splicing & splicing & NA & 0.001103 \\
\hline P29 & 4 & 8552.05 & TP53_p.H179R & exonic & nonsynonymous SNV & 0.008401 \\
\hline P29 & 4 & 8552.05 & PIK3CA_p.H1047L & exonic & nonsynonymous SNV & 0.026549 \\
\hline P29 & 4 & 8552.05 & CDKN2A_splicing & splicing & NA & 0.006991 \\
\hline P30 & 3.5 & 3895.89 & ARID1A_p.S593F & exonic & nonsynonymous SNV & 0.001214 \\
\hline P30 & 3.5 & 3895.89 & BCL11B_p.K875M & exonic & nonsynonymous SNV & 0.000997 \\
\hline P30 & 3.5 & 3895.89 & TP53_p.G154V & exonic & nonsynonymous SNV & 0.00984 \\
\hline P30 & 3.5 & 3895.89 & TP53_p.D61G & exonic & nonsynonymous SNV & 0.001346 \\
\hline P30 & 3.5 & 3895.89 & SLIT2_p.P1066T & exonic & nonsynonymous SNV & 0.001821 \\
\hline P31 & 3.5 & 8195.57 & GRIN2A_p.R244S & exonic & nonsynonymous SNV & 0.001692 \\
\hline P31 & 3.5 & 8195.57 & TP53_p.C238W & exonic & nonsynonymous SNV & 0.001038 \\
\hline P31 & 3.5 & 8195.57 & KEAP1_p.S233N & exonic & nonsynonymous SNV & 0.001346 \\
\hline P31 & 3.5 & 8195.57 & FAM135B_p.D555H & exonic & nonsynonymous SNV & 0.001853 \\
\hline P37 & 3.5 & 10177.1 & & & & \\
\hline
\end{tabular}

SNV, single nucleotide variant; NA, not applicable. 


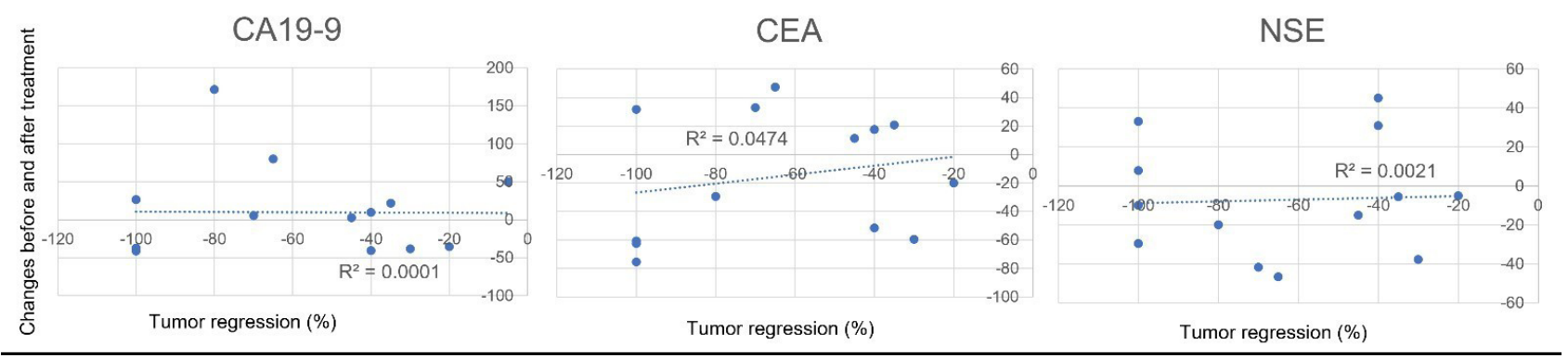

Figure S3 Correlation between pathological response and clinically used tumor biomarkers. CA19-9, serum carbohydrate antigen 19-9; CEA, carcinoembryonic antigen; NSE, neuron-specific enolase. 

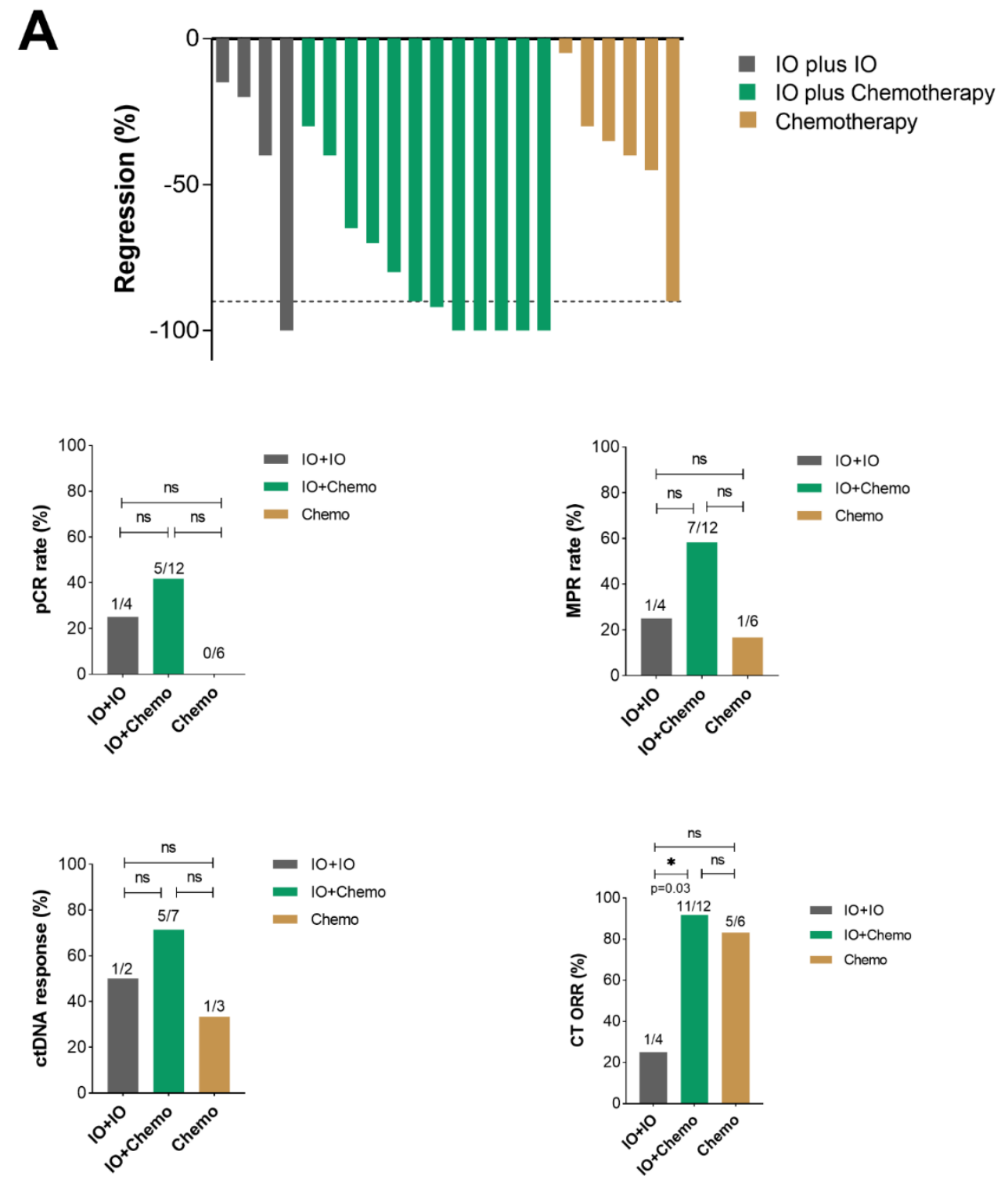

Figure $\mathbf{S} 4 \mathrm{ctDNA}$ change and pathological response in patients receiving different neoadjuvant regimens. (A) Tumor regression of patients treated with immunotherapy plus chemotherapy (IO+Chemo), IO+IO, and Chemo alone. (B) The pathological complete response (pCR), major pathological response (MPR), ctDNA response rates, and computed tomography (CT) response (objective response rate [ORR]) in each treatment group. ctDNA response was defined as relative delta mean variant allele fraction (R $\Delta$ mean VAF) to depict dynamic changes of ctDNA upon neoadjuvant therapy. To aim for a maximum sum of specificity and sensitivity for predicting pathologic response, the threshold for defining responders was determined to 0.98. ORR was defined as the frequency of patients who have had achieved complete response (CR) or partial response (PR) at two consecutive CT assessment at least 4 weeks apart. *, $\mathrm{P}<0.05$. IO, immunotherapy; Chemo, chemotherapy; IO+IO, dual immunotherapy; ctDNA, circulating tumor DNA; pCR, pathological response; MPR, major pathological response; CT, computed tomography; ORR, objective response rate. 


\section{Before surgery}
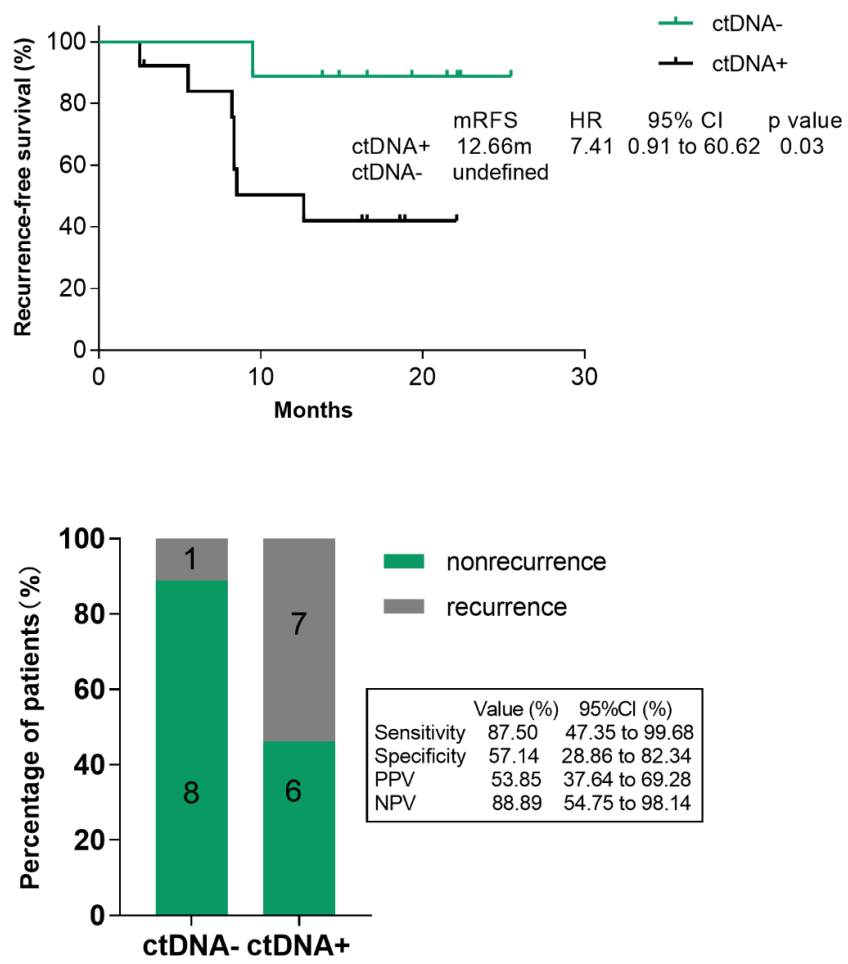

Figure S5 Absence of ctDNA after neoadjuvant therapy correlates with better recurrence-free survival (RFS). Kaplan-Meier survival curves depicting RFS of patients having had ctDNA detection after neoadjuvant therapy (within one week before surgery). HR, hazard ratio; CI, confidence interval; PPV, positive predictive value; NPV, negative predictive value. ctDNA-, without detectable mutation; ctDNA+, with detectable mutation.

A

One week after surgery (IO+Chemo)

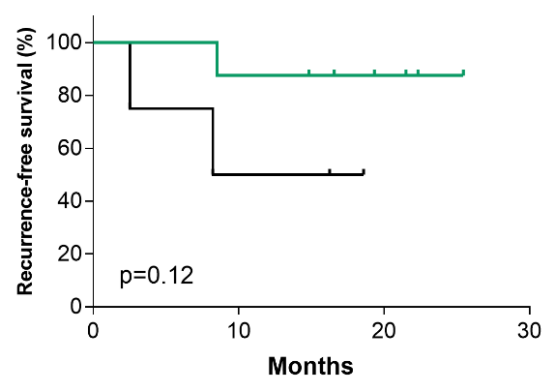

B

Three months after surgery (IO+Chemo)

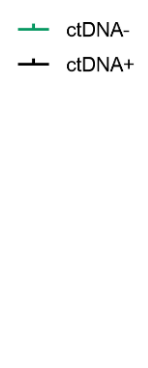

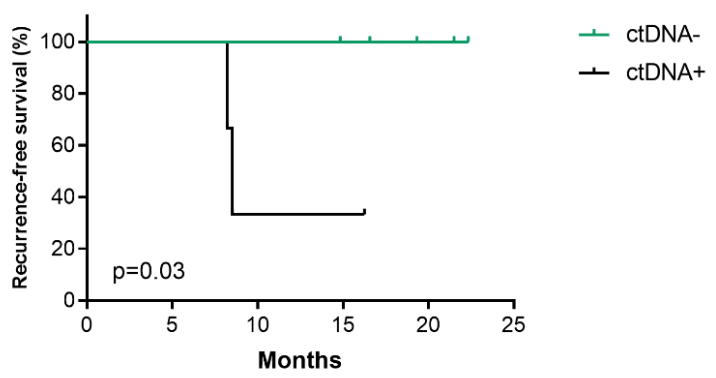

Figure S6 Correlation between postoperative ctDNA and recurrence-free survival in patients treated with immuno-chemotherapy. KaplanMeier survival curves depicting RFS of patients having had ctDNA detection at one week (N=22) (A) and three months (N=16) (B) after surgery. Patients who had received neoadjuvant nivolumab plus chemotherapy were analyzed. IO, immunotherapy; Chemo, chemotherapy; RFS, recurrence-free survival; ctDNA-, without detectable mutation; ctDNA+, with detectable mutation. 
Table S4 Univariate and multivariate analyses of prognostic factors for recurrence-free survival

\begin{tabular}{|c|c|c|c|c|c|c|}
\hline Factors & \multicolumn{3}{|c|}{ Univariate Cox } & \multicolumn{3}{|c|}{ Multivariate Cox } \\
\hline Age (<60 vs. $\geq 60$ years) & 1.96 & $0.40-9.72$ & 0.41 & & & \\
\hline Sex (male vs. female) & 1.7 & $0.34-8.47$ & 0.52 & & & \\
\hline Smoking (no vs. yes) & 0.19 & $0.05-0.83$ & 0.01 & 0.24 & $0.05-1.17$ & 0.077 \\
\hline Disease stage (I \& II vs. III) & 0.35 & $0.08-1.46$ & 0.15 & & & \\
\hline \multicolumn{7}{|l|}{ Neoadjuvant therapy } \\
\hline IO plus IO vs. IO plus Chemo & 0.27 & $0.04-1.66$ & 0.16 & & & \\
\hline IO plus IO vs. Chemo & 0.51 & $0.08-3.10$ & 0.46 & & & \\
\hline
\end{tabular}

${ }^{*}$, ctDNA presence or absence in patient's blood sample at one week after surgery. HR, hazard ratio; 95\% Cl, confidence interval; IO, immunotherapy; Chemo, chemotherapy; Sq, lung squamous cell carcinomas.

\section{References}

44. NCCN Clinical Practice Guidelines in Oncology. Non-Small Cell Lung Cancer. Version 4. 2021 [database on the Internet]. National Comprehensive Cancer Network (NCCN). 2021. Available online: www.nccn.org.

45. Newman AM, Bratman SV, To J, et al. An ultrasensitive method for quantitating circulating tumor DNA with broad patient coverage. Nat Med 2014;20:548-54.

46. Ding L, Getz G, Wheeler DA, et al. Somatic mutations affect key pathways in lung adenocarcinoma. Nature 2008;455:1069-75.

47. Youn A, Simon R. Identifying cancer driver genes in tumor genome sequencing studies. Bioinformatics 2011;27:175-81. 